\title{
Fiber-Optic Sensors for Explosives Detection
}

\author{
Jianjun $\mathrm{Ma}^{*}$ and Wojtek J. Bock
}

Centre de Recherche en Photonique, Département d'informatique et d'ingénierie, Université du Québec en Outaouais, P. O. Box 1250, Hull Station, Gatineau, Québec J8X 3X7, Canada

\begin{abstract}
As one of the most enduring hot topics, explosives detection commands world-wide interest among scientists across universities and research institutes. Abundant research achievements pertaining to this subject have beeneported. Various choices of commercial and military-grade detection units are available for deployment. Yet, airport security and the continuing threat of improvised explosive devices (IEDs) demand more advanced solutions for the detection of trace explosive materials. Among the most valued are those capable of detecting trace explosive vapors in open air, which have also presented the most technically difficult challenges confronting researchers.

This paper is devoted to the detection of trace vapor explosive materials using fiber-optic sensors and lab-on-a-fiber (LOF) devices. To offer readers an overall picture of the subject, recent technical progress is reviewed by covering all available techniques. The rapid evolution of laser-induced breakdown spectroscopy (LIBS) and Raman spectroscopy from benchtop instruments to field-deployable and portable units is emphasized as these techniques are widely used for detection of trace explosives and strongly related to fiber-optic sensing. The major part of this paper, however, will discuss our LOF devices coated with amplifying fluorescent polymer (AFP) films for the detection of trace vapor explosive materials. Various LOF devices employing low quantum yield (QY) AFPs will be described and evaluated. The crucial factors affecting the performance of LOF devices will be explored. On-line trace vapor pre-concentration and gas chromatography (GC) will be briefly discussed due to the indispensability of this approach for the fast and reliable on-line detection of trace vapor explosive materials.
\end{abstract}

Keywords: Fiber-optic sensor, Explosive detection, Explosive vapor detection, Amplifying fluorescent polymer, Lab-on-afiber.

\section{INTRODUCTION}

\subsection{Lab-on-a-Fiber (LOF): A Special Class of Fiber- Optic Sensor}

Traditionally, a fiber-optic sensor (FOS) is either intrinsically or extrinsically based, depending on its mechanism of operation. For an extrinsic FOS, the fiber or fibers are used for optical signal delivery only. Free-space or micro-opticsbased components constitute the sensing section. One example of an extrinsic FOS is the fiber-optic displacement sensor comprising a bifurcated or a coaxial fiber bundle with a movable mirror placed a short distance in front. For an intrinsic FOS, the fiber or fibers serve both sensing and signal delivery purposes, with typical examples being fiber Bragg grating (FBG) sensors, long-period grating (LPG) sensors, and fiber-optic distributed sensors based on either Raman or Brillouin scattering.

The FOS family has grown rapidly since its first appearance in the mid-1970s. The classifications of FOSs as intrinsic/extrinsic or physical/chemical are still rather crude and do not adequately reflect the particular features of a sensor architecture that may be significant for a particular

\footnotetext{
*Address correspondence to this author at the Centre de recherche en photonique, Département d'informatique et d'ingénierie, Université du Québec en Outaouais, P. O. Box 1250, Hull Station, Gatineau, Québec J8X 3X7, Canada; Tel: 1-819-5953900x1613; Fax: 1-819-7731638;

E-mail:ma.jianjun@uqo.ca,wojtek.bock@uqo.ca
}

application. For example, a FOS with a mini sensing element directly attached to the fiber has much more to offer in terms of real applications than its bulkier counterparts. In recognition of this fact, we have proposed the lab-on-a-fiber (LOF) concept $[1-3]$ as a specific category restricted to FOSs featuring millimeter-scale sensing elements. One or more regular laboratory functions or components could be downsized to fit into the tiny space of an LOF to meet the needs of a specific application.

Originally inspired by the lab-on-a-chip (LOC) concept, the LOF device has potential functions that run across many disciplines, from chemistry and physics to optics. The fibers in the LOF are substrates that are integrated with optical elements such as a light-source shaper, a mirror, a lens, an optical signal collector and an enhancer. These components are directly built on the fiber in various forms depending on the application, or they are created by shaping fiber itself. This not only ensures the tiny size of the LOF device but also maximizes its robustness, another major goal for the LOF proposition. Functions associated with chemical processes could also be included by attaching them directly to the fiber using appropriate techniques.

\subsection{Impact of New Optical Components on FOSs for Ex- plosives Detection}

Many different techniques are now available for explosives detection. Steady advances have resulted from incorporation of newly commercialized key components that are 
smaller than their predecessors and outperform them. Laserinduced breakdown spectroscopy (LIBS) systems, for example, have evolved from large benchtop analytical instruments to portable FOSs for field operation. This is clearly illustrated by the contrast between the LIBS2500 [4] shown in (Fig. 1(a)) and the fiber-coupled LIBS sensor in Fig. 1(b) [5]. LIBS2500 comprises a Nd:YAG laser, a cooling system, a free-space optics focusing assembly, a sample imaging system and a large sampling chamber, constituting a large benchtop analytical instrument. A seven-fiber bundle conveys the signal light from the sample to a spectrometer array that covers a wide wavelength band from UV to NIR. In contrast, the LIBS system in (Fig. 1(b)) has been engineered to fit into a $0.7 \times 0.7 \times 0.7 \mathrm{~m}$ cube by employing a dedicated master oscillator fiber amplifier (MOFA). The bulky laser system is replaced by a piece of fiber (MOFA) and few other small components. Outside the cube is a wand that encloses a fiber bundle with a mechanical shutter and a gas flow system. The entire system is lightweight, portable and ready for field operation.

Another example of a portable fiber-optic biosensor, as illustrated in (Fig. 2), is from the research group in the Naval Research Lab and has been commercialized by Research International Inc. [6,7]. Both Analyte 2000 and Raptor systems are capable of monitoring four channels simultaneously, Raptor being the automated and portable version designed for a battlefield environment. Raptor's portability can be partially attributed to technical progress made in LED light sources in terms of power level, stability, lifetime and cost. Our LOF-based systems, as we shall see later, are even lighter in weight and highly portable, owing to the small footprint of the Ocean Optics USB 2000 spectrometer and the LED source with its built-in SMA connectors.

\section{OVERVIEW OF EXPLOSIVE MATERIALS AND METHODS OF DETECTING THEM}

\subsection{Overview of Explosive Materials Associated with Terrorist Activities}

Although several hundred explosive materials are officially listed under the United States Code [8], most commercial explosives are organic compounds containing $-\mathrm{NO}_{2},-$
$\mathrm{ONO}_{2}$ and $-\mathrm{NHNO}_{2}$ groups, with TNT, nitroglycerin, $\mathrm{HMX}$, PETN and nitrocellulose as the typical examples. However, inorganic species have also been found in connection with terrorist activities, including inorganic nitrates, chlorates or perchlorates, and unstable peroxide groups such as triaceton triperoxide (TATP). Five of the explosives of greatest interest are listed in (Table 1), along with their possible vapor components [9]. Fig. (3) illustrates the molecular architectures of nine of the most widely used nitroaromatic explosive materials.

\subsection{Overview of Methods for Detecting Explosive Mate- rials}

\subsubsection{Methods Based on Spectroscopy}

Spectroscopy is one of the most important techniques for explosives detection. Among the principles that are central to detection systems for forensic investigation, airport security and monitoring of environmental pollution are ion mobility spectroscopy (IMS), mass spectroscopy (MS), LIBS and Raman spectroscopy. Other techniques such as Terahertz (THz) spectroscopy, infra-red spectroscopy and cavity ringdown spectroscopy (CRDS) are of continuing interest to researchers due to their unique advantages.

\subsubsection{Ion Mobility Spectroscopy}

Ion mobility spectroscopy (IMS) is a mature technique routinely applied for both qualitative and quantitative sample analysis featuring very low detection limits [10,11]. IMS vaporizes and ionizes samples at atmospheric pressure before sending them to the drift tube. The mass of the ions is directly associated with the drift times, based on which the mass/charge ratio can be determined. The components within the sample can also be identified through comparison with known standards.

Miniaturization and portability have been priorities to support field deployment of IMS apparatus. Technical barriers, and thus major targets of research efforts, include the length of the drift tube, and the size of the ionization source as they affect the sensitivity of the apparatus. Competitive cost is also an important factor driving the solutions to these barriers.

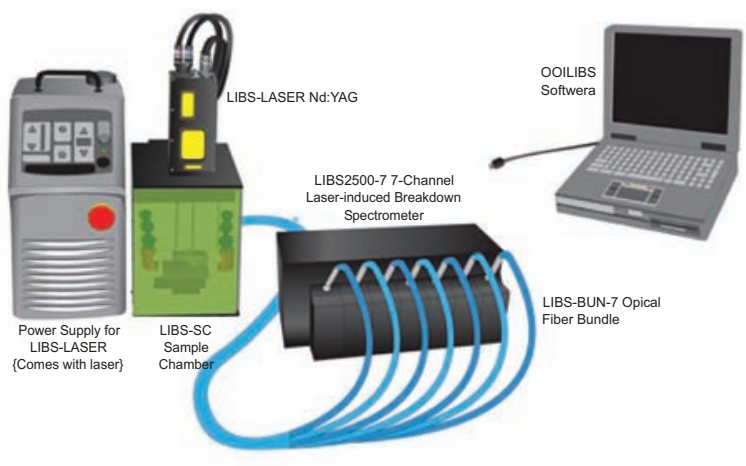

(a)

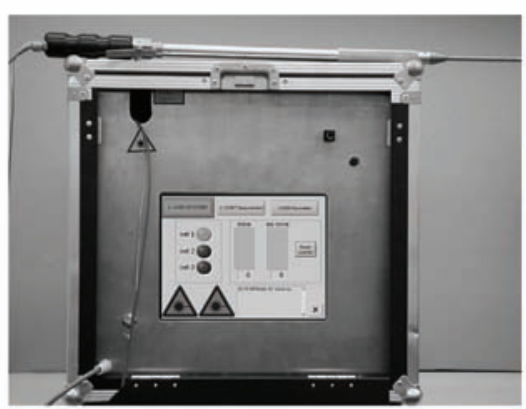

(b)

Fig. (1). Two LIBS systems for explosives detection. (a) Benchtop LIBS2500 from Ocean Optics ${ }^{\mathrm{TM}}$. (b) Fiber-coupled portable LIBS sensor $[5]$. 


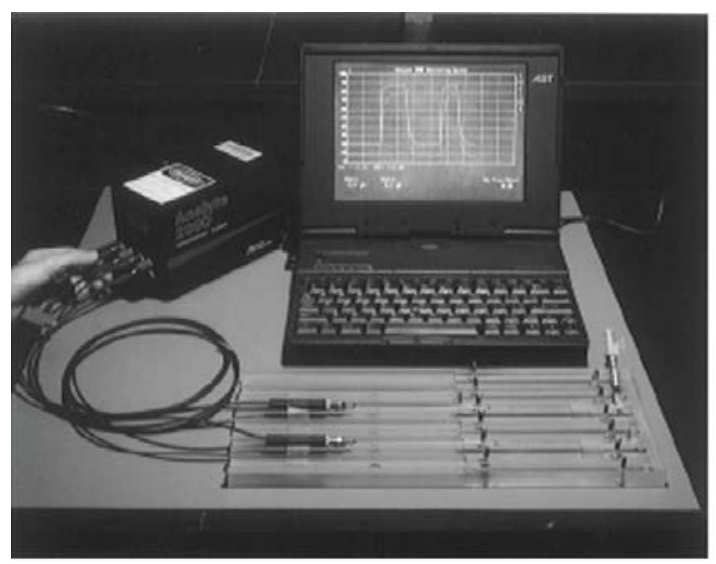

(a)

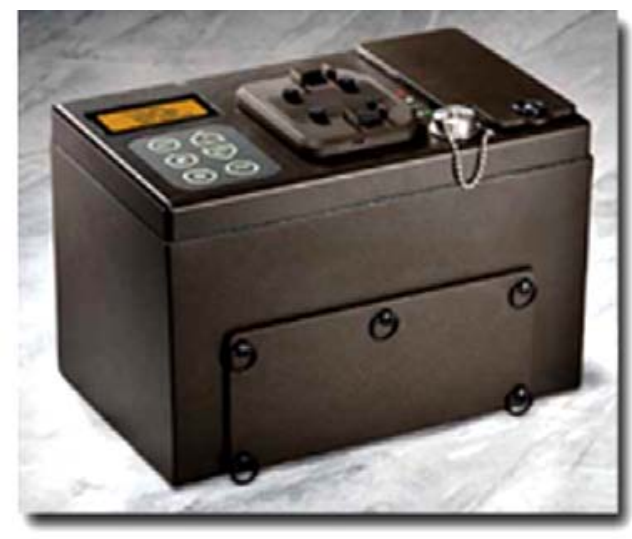

(b)

Fig. (2). Fiber-optic biosensors developed by Naval Research Lab in collaboration with Research International Inc. (a) Analyte 2000 portable fiber-optic biosensor. (b) User-friendly Raptor model for battlefield environment.

Table 1. The Five Explosives of most Interest

\begin{tabular}{|c|c|c|}
\hline Explosive & Formulations and other Uses & Vapor Components \\
\hline \hline TNT & Military grade castings, Composition B & 2NT, 4NT, 24DNT, 26DNT \\
\hline RDX & C-4, Semtex, Composition B & Cyclohexanone, hexamine, taggants \\
\hline HMX & PBXs, rocket propellant, HE & Taggants \\
\hline PETN & DETAsheet, primacord & Acetone, TATP \\
\hline TATP & Terrorist use & \\
\hline
\end{tabular}

TNT2,4,6-trinitrotoluene, $R D X$ 1,3,5-trinitroperhydro-1,3,5-triazine, $H M X$ 1,3,5,7-tetranitroperhydro-1,3,5,7-tetrazocine, PETN pentaerythritol tetranitrate, $P B X$ polymer-bonded explosive, $H E$ high explosive, $2 N T$ 2-nitrotoluene, 4NT 4-nitrotoluene, 24DNT, 2,4-dinitrotoluene, 26DNT 2,6-dinitrotoluene, TAPT tricetone triperoxide

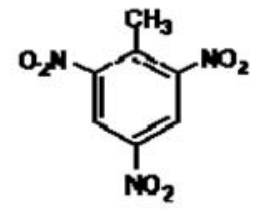

(a)

2, 4, 6-trinitrotoluene (TNT)

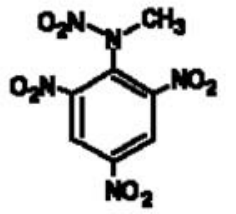

(d)

1,3,5-trinitrobenzene (TNB)<smiles>O=[N+]([O-])N1CN([N+](=O)[O-])CN([N+](=O)[O-])C1</smiles>

(g)

Cyclotrimethylenetrinitramine (RDX)

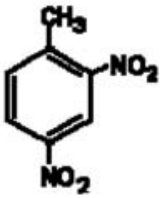

(b)

2, 4-dinitrotoluene (DNT)

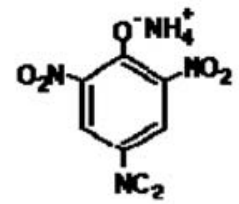

(e)

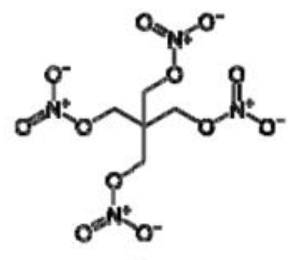

(h)

Pentacrythritol tetranitrate (PETN)<smiles>O=[N+]([O-])c1cc([N+](=O)[O-])c(O)c([N+](=O)[O-])c1</smiles>

(c)

2, 4, 6-trinitrophenol (TNP) (Picric acid)<smiles>O=[N+]([O-])c1cc([N+](=O)[O-])cc([N+](=O)[O-])c1</smiles>

(f)

2,4,6-trinitrophenylmethylnitramine

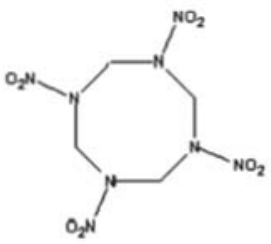

3,5,7-tetranitroperhydro-1,3,5,7-tetrazocine (HMX) (i)

Fig. (3). Chemical structures of some common nitroaromatic explosive materials. 


\subsubsection{Mass Spectroscopv}

MS identifies an explosive material according to its mass-to-charge (m/e) ratio, which is achieved by analysing the chemical composition through time or geometric separation. Mass spectroscopy may be realized by quadrupole, ion trap, time-of-flight (TOF) or tandem-based techniques (MS/MS) [12].

As with IMS, size and expense are important considerations. Pre-concentration of samples is crucial at present, and much research is directed to eliminating this step by improving the techniques for introducing samples into the MS apparatus and thus enhancing the sensitivity towards the material of interest [11].

When atmospheric pressure chemical ionization (APCI) is employed, MS can selectively detect nitroaromatic explosives [13]. APCI with reversed gas-flow improves the ionization of nitro-compounds, and used in a tandem mass spectrometer has demonstrated the ability to detect $3 \mathrm{pgL}^{-1}$ TNT in samples collected on a swab directly from the skin of a hand that has handled explosive material [14].

One early portable mass spectrometer demonstrated the ability to rapidly detect explosive compounds in situ from a variety of surfaces, with a detection limit of less than 1 nanogram (ng) for RDX on paper [15].

In another development, GC has been combined with MS (GC-MS), bringing together the separation ability of GC with the sensitivity and specificity of MS for explosives detection. GC-MS-based units have been widely deployed in U.S. airports since the $9 / 11$ attack. A typical system combines high-speed GC with ion-mobility MS, enabling it to detect a range of explosives, including $\mathrm{C} 4$, Semtex, peroxides, TNT and nitrates at nanogram levels in a matter of seconds.

\subsubsection{Terahertz Spectroscopy}

$\mathrm{THz}$ spectroscopy is highly valued due to the deep penetration of its radiation into non-polar dielectric materials such as wood or leather, which is enabled by its far infra-red operation from 0.1 to $10 \mathrm{THz}$ (the sub-millimeter wavelength band). The photon energy of the THz wave is $\sim 10^{-6}$ times less than that of an X-ray photon and the fingerprints of most explosive materials can be found within this $\mathrm{THz}$ range. Apparatuses operating in this band are readily available, giving THz spectroscopy convenient access to prospective sensor and imaging agents for the identification of concealed explosive substances.

$\mathrm{THz}$ spectroscopy has been investigated as a means of detecting the vapor signature of explosives $[9,16,17]$. Existing issues for this technology include frame-rate speed, signal loss and the required excitation power, which depends on the sample distance [18]. Other research has focused on its ability to detect not only explosives, but also concealed weapons and other objects within luggage or beneath fabric. RDX on an uneven surface whether exposed or covered by various materials has also been studied, showing a consistent fingerprint at $0.82 \mathrm{THz}$ [19]. Perhaps most significantly, the absorption spectra of 17 explosives and their related compounds have been examined, in work that revealed their characteristic absorption features in the 0.1-2.8 $\mathrm{THz}$ range and thus indicates the prospect of forming a database of explosives [20].

\subsubsection{Infra-Red Spectroscopy}

Infra-red spectroscopy apparatus extracts the fingerprints of the targeted species by examining the absorption spectrum. In principle this spectrum could be obtained by simply passing an infra-red beam through the sample. Yet, the traditional infra-red spectroscopy has a slow response due to the time needed for wavelength scanning over a dispersed target. The solution is Fourier-transform infra-red (FTIR) spectroscopy, which simultaneously scans all concerned wavelengths and thus significantly reduces the time required for investigation.

A fiber-optic probe has been used in conjunction with reflection/absorption FTIR spectroscopy to detect TNT, PETN or DNT and HMX and has demonstrated low detection limits of $160 \mathrm{ng} / \mathrm{cm}^{2}, 220 \mathrm{ng} / \mathrm{cm}^{2}$ and $400 \mathrm{ng} / \mathrm{cm}^{2}$, respectively [21]. Despite its use of the fiber-optic probe, this FTIR system is a high-end bench-top instrument.

\subsubsection{Cavity Ring-Down Spectroscopy}

The key element of CRDS is a resonant cavity filled with either air or sample species, such as explosive vapors. Resonant radiation is established by launching a laser into the cavity with a wavelength matching the cavity modes. When sufficient radiation has established, the laser is turned off, and the exponential decay of the light over time can be measured, the result being the absorption fingerprint of the gas sample in the cavity. Very high sensitivity can be achieved due to the multiple reflections between mirrors. However, even with the high sensitivity of CRDS, identifying an explosive from its vapor is still not a simple matter, as evidenced by the research report showing that CRDS has high sensitivity but poor selectivity in the UV range versus low sensitivity but better selectivity in the near infra-red range when detecting 2,4-DNT and 2,6-DNT [22]. Nonetheless, CRDS is a spectroscopic tool offering exceptionally high sensitivity to trace explosive vapor in air.

\subsubsection{Methods Based on an Olfactory Sensor or "Sniffer"}

The idea for an olfactory sensor or a "sniffer" originated from the superb ability of canine noses to locate explosives. Despite rapid advances in technique, so far only a few highend artificial instruments such as those using IMS can compete with dogs in terms of detection limits. Besides, dogs could be trained to sniff a virtually unlimited range of explosive types as well as non-explosive species. They have proven their value for the detection of dangerous chemicals and for sensing at problematic or unsafe scenes. Of course, as living detectors, dogs have to be trained by professionals through a costly and time-consuming process. They may also get tired after long hours of work and may be confused by a mixture of scents.

Inspired by the sniffing capability of a dog's nose, the artificial equivalent - the electronic nose - emerges as a unique category of explosives detectors. The key element of the electronic nose is the chemical sensor or the sensor array that interacts with explosive vapors, each individual sensor responding differently to the vapors. An olfactory array could be combined with a pattern recognition system such as 
an artificial neural network to determine the specific analyte [23].

\subsubsection{Methods Based on Chemical Sensors}

Chemical sensors operate by detecting chemical reactions in the presence of explosive material leading to changes in color or conductivity, or to fluorescence turn-off $[24,25]$. Such devices include electrochemical and immunochemical sensors as well as sensors based on luminescence.

Electrochemical sensors detect explosive materials by measuring the voltage, current or conductivity of the signals they emit. They offer the advantages of fast response, low cost, high sensitivity and potential for miniaturization [26]. By responding to redox chemical reaction, they can detect not only nitroaromatic explosive materials, but also inorganic explosive materials such as TATP that contain no nitro groups, have low UV absorption and do not fluoresce at all [27].

Research activities based on electrochemical sensing have delivered many promising results. Examples include the high sensitivity achieved by enlarging the surface area of the electrode $[28,29]$, the selective detection of nitroaromatics such as TNT using a special sensing layer attached to the electrode [30], and the fast detection of peroxide-based explosives [31] and TATP [32]. LOC technology has also been studied to explore its feasibility as a fast, simple and cheap alternative to lab-based techniques [33]. A wearable sensor built into fabric has been also investigated and has successfully detected nitroaromatic compounds (NACs) [34].

Immunochemical sensors can identify species with high selectivity based on antibody-antigen reaction. This fact, together with the possibility of making antibody for a specific sample target, has triggered extensive research into the application of this approach for explosives detection. For example, the portable fiber-optic biosensors in (Fig. 2) apply competitive immunoassay to fiber-optic probes functionalized by antibody to identify explosive substances. The Analyte 2000 biosensor is able to detect TNT and RDX explosives with a detection limit of $6 \mathrm{ng} / \mathrm{ml}$ [7].

Surface plasmon resonance (SPR) is a powerful tool for the detection of chemical and biological events due to its extraordinary sensitivity to variation in the refractive index, achieved by coating a thin layer of precious metal such as gold or silver on the surface of the waveguide. The refractive index change on the metal surface can then be detected by observing the shift of the SPR resonance angle.

Immunochemical sensors have been widely adopted for this application to immobilize antibody on the waveguide surface in order to capture and bind the sample molecules. Proper selection of the immunochemical sensor for the SPR apparatus is crucial. Research indicates that a larger SPR signal is strongly associated with the binding event of large molecules, while the SPR signal generated by molecules of an explosive is usually weak owing to the small size of the molecules. A proven solution is to introduce the indirect competitive binding effect to the SPR system operation $[35,36]$.

Another popular chemical sensor is based on luminescence, mainly on fluorescence. The appearance of the fluo- rescent light may directly relate to the presence of explosives, whether the light emits by itself, or through chemical reaction. A fluorescent material, on the other hand, is able to detect explosive compounds via fluorescence quenching or turn-off upon contact with explosive molecules. A comprehensive review of this technique is given in reference [37]. While AFP sensory material belongs to this chemical sensor category, we will describe it in Section IV due to its significance for our LOF-based system based on its extraordinary amplification mechanism.

\subsubsection{Methods Based on Nanotechnology}

Materials in nanoscale behave substantially different from their bulky counterparts, which may generate unusual but desirable features not offered by large-size materials. For this reason, nanotechnology has attracted huge research interest in recent years. For example, carbon nanotubes enhance sensitivity owing to the large surface area. They are mechanically strong, have electrocatalytic effects, and possess excellent flexibility and conductivity. Nanoparticles demonstrate a number of optical effects that significantly enhance the sensitivities of SPR and surface-enhanced Raman spectroscopy apparatuses. The detection of explosive compounds such as PETN, RDX and TNT based on nanoparticles has been investigated. Response time for TNT vapor is $\mathrm{pg} / \mu \mathrm{g} \mathrm{MIP} /$ minute by employing molecularly imprinted polymers (MIP) and quartz crystal microbalance (QCM) [38]. By modifying the glassy carbon electrodes with multi-walled carbon nanotubes to increase the surface area for accumulation of TNT, it was found that TNT could be detected down to the sub- $\mu \mathrm{g} / \mathrm{L}$ level and an LOD of $0.6 \mu \mathrm{g} / \mathrm{L}$ [39].

\subsubsection{Methods Based on Quartz Crystal Microbalance}

Quartz crystal microbalance (QCM) has a resonance frequency that may be disturbed by adding a mass to it, or by removing a mass, the frequency change indicating the presence of the targeted substance. The density of this mass may be down to the level of below $1 \mu \mathrm{g} / \mathrm{cm}^{2}$. By functionalizing the QCM with specific coating to enable a process resembling the immobilizing/binding events of antibody-antigen, selective detection with high sensitivity is possible. One example shows that a QCM could detect TATP vapor in nitrogen with a lower detection limit at $1 \mathrm{ppm}(6.6 \mu \mathrm{g} / \mathrm{L})$ [40].

\section{OVERVIEW OF FIBER-OPTIC SENSORS FOR EX- PLOSIVES DETECTION}

\subsection{LIBS Spectroscopy: From Bench-Top Equipment to Field-Deployable and Portable Systems}

In a LIBS system, the laser launches a high-power pulse to vaporize a tiny quantity of sample material and generate a plasma plume. Light emitting from this plasma plume is collected by a fiber bundle for analysis, which reveals the characteristics of the sample. Advantages of the LIBS system include real-time analysis, the potential of miniaturization for field work, stand-off operation and the fact that no sample preparation is needed. The recent inclusion of LIBS in the ChemCam instrument suite onboard the NASA Mars Science Laboratory rover for extraterrestrial applications is evidence of the outstanding ability of LIBS as a spectroscopic-based technology [41]. 
The unique advantages of LIBS provided the stimulus for recent major research efforts by the U.S. Army Research Lab (ARL) to develop field units to meet the pressing demands from the military for stand-off explosives detection capability at a distance of $\sim 100 \mathrm{~m}$, which is safe for both screeners and equipment $[42,43]$. Fig. 4(a) illustrates the principle of operation of this open-path configuration LIBS system while the photograph in Fig. 4(b) shows their double-pulse standoff system ST-LIBS developed in collaboration with two commercial companies [44]. Fibers are used to receive the light collected by free-space optics and send it to the spectrometers for analysis, as shown in Fig. 4(a). ARL and the two companies have built several prototypes that can be mounted on a vehicle for $>100 \mathrm{~m}$ stand-off distance as in Fig. 4(c) or ranging from 10 to $100 \mathrm{~m}$. They have also built the man-portable prototype for routine hazardous material detection shown in Fig. $\mathbf{4}(\mathbf{d})$ based on the same configuration as in Fig. 4(a).

While this man-portable prototype appears similar to the prototype illustrated in Fig. 1(b), in fact the two devices use substantially different techniques and approaches to achieve the portability of their systems. Although the open-path configuration offers great flexibility with a stand-off distance, hard-to-reach areas with awkward objects blocking the light path needed an alternative solution. Applied Photonics Ltd. in the U.K. developed a $75 \mathrm{~m}$ fiber-optic LIBS system for remotely determining the copper content of a stainless steel superheater bifurcated tubing within the pressure vessel of a nuclear power station [45]. Fig. 5(a) illustrates the principle of operation and Fig. 5(b) shows a photograph of the installation of this system. This solution did not use either freespace optics (Fig. 4(a)) or any active fiber (Fig. 1(b)) for high-power laser delivery. Instead, this system uses a $75 \mathrm{~m}$ $550 \mu \mathrm{m}$ core fiber for both laser power delivery and signal collection.

\subsection{Raman Spectroscopy: From Bench-Top Equipment to Field-Deployable and Portable Systems}

Raman spectroscopy apparatus identifies explosives by detecting changes in the state of vibrational energy under laser excitation. The unique fingerprint of an explosive molecule is revealed by the vibration of its chemical bond since it is related not only to the composition of materials but also to the molecular structure of each constituent. Even a trace amount of explosive residue can provide measurable Raman-scattered light. It has been demonstrated that a fieldprototype system can detect and identify sub-milligram quantities of explosives at a distance of up to $30 \mathrm{~m} \mathrm{[46].}$

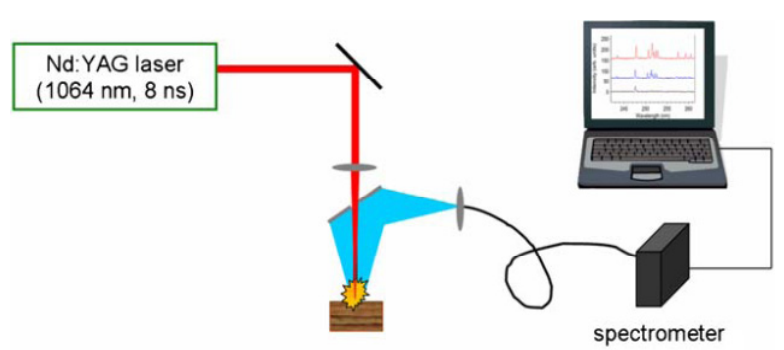

(a)

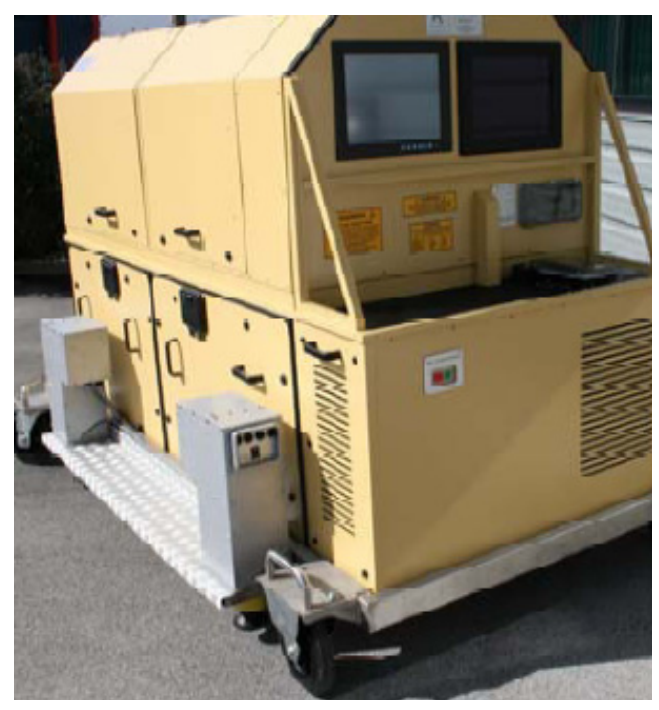

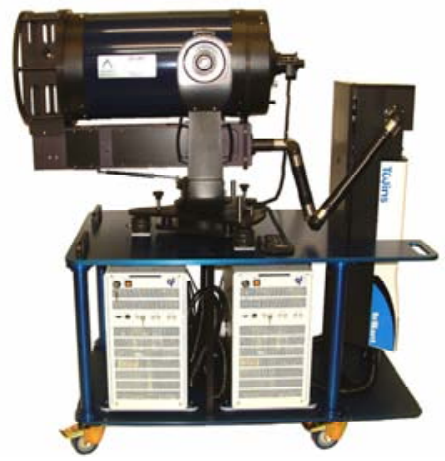

(b)

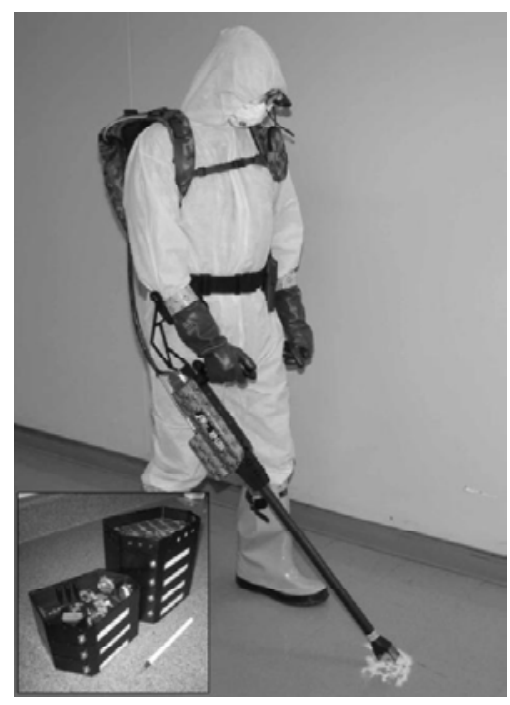

Fig. (4). The LIBS system developed by ARL. (a) Principle of operation of the open-path LIBS system; (b) The stand-off LIBS system (STLIBS) developed by ARL in collaboration with Applied Photonics Ltd. and Ocean Optics Inc. (c) The open-path LIBS prototype mounted on a vehicle for stand-off distance $>100 \mathrm{~m}$; (d) Man-portable LIBS prototype for the detection of IEDs. 


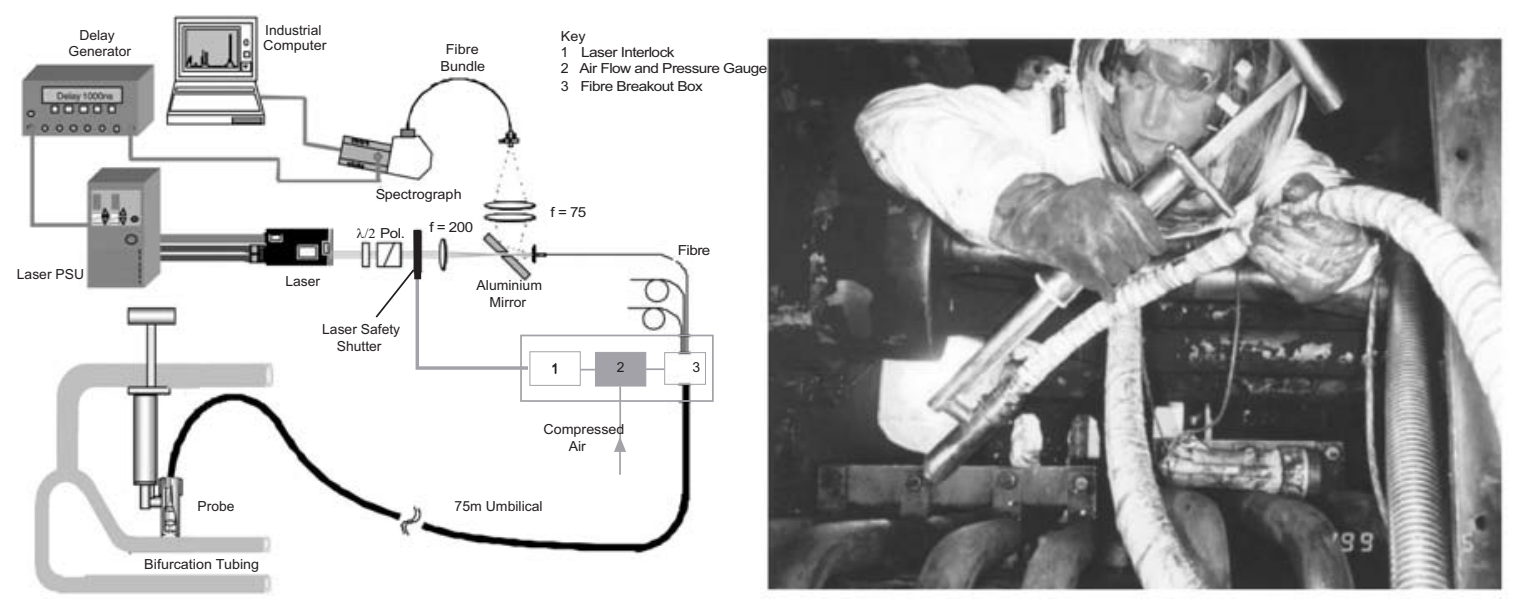

Fig. (5). A LIBS system with a $75 \mathrm{~m}$ all-fiber probe developed by Applied Photonics Ltd. (a) Principle of operation; (b) Deployment of the all-fiber LIBS probe inside a superheater.

Moreover, the Raman spectra of explosives and other substances are available as commercial databases and in the open literature [47].

A number of portable Raman-spectroscopy-based devices are commercially available and can identify small amounts of explosives in the field. Some of these use fiber-optic probes for remote detection [48]. However, the weak Raman scattering signal is a major barrier to improving the performance of portable systems. In addition, these portable systems only work well with white explosives and have proven problematic with aged or colored substances because of the strong background fluorescence and the risk of explosion due to the strong absorption of excitation power [48].

The Swedish Defence Research Agency studied a standoff Raman-based system for detecting various IEDs at distances of 20, 30 and $55 \mathrm{~m}$ under varying weather conditions from snow to sun and at temperatures from $22^{\circ} \mathrm{C}$ to $-8^{\circ} \mathrm{C}$. The experiments were carried out by keeping the instruments inside buildings and the bulk sample in the open environment. While weather was found not to affect the results, neither the interfering fluorescence from the container nor the ability to detect trace amounts of explosive substance were investigated [49].

\subsection{LIBS-Raman System: Combination can Reduce False Alarms}

Since both LIBS and Raman spectroscopy can identify explosives and discriminate them from other materials at stand-off distances, research has been done into combining the two techniques. It was found that the molecular vibrational information revealed by Raman spectroscopy could complement the elemental compositions determined by LIBS and reduce the probability of false alarms when detecting trace-level explosive materials [50].

\subsection{Explosives Detection Using a Functionalized Large- Core Fiber Taper}

An evanescent-wave (EW) based device is often a preferred tool for sample assay, with the one illustrated in
(Fig. 2) being a successful example [6, 7]. Fig. (6) shows the key element of this system, a modified large-core fiber taper. This taper preserves the merits of the regular fiber taper that is able to simultaneously enhance EW excitation power level and improve EW signal power collection efficiency via the mode-conversion process enabled by the geometry of the taper. Owing to this mode-conversion mechanism, the taper, while short, efficiently couples the captured EW signal light to better confined lower-order modes and thus diminishes interference due to fiber bends and defects on the corecladding interface.

The structure in (Fig. 6) represents a different design. It employs a regular cylinder waveguide for sampling, tailed with a short tapered section called a "concentrator". This increases the excitation power level even further while maintaining the same mode-conversion feature as the original design as long as the cylindrical section is short. The Raptor model in Fig. 2(b) uses a disposable plastic coupon containing four such tapers shaped by injection mold. These tapers are functionalized with the desired chemistry such as antibody-antigen and inserted into the coupon. To run an assay, the user simply inserts a coupon and presses the Run Assay key. Assays take 10-15 minutes with a sensitivity of 1 to 10 parts per billion (ppb) depending on the analyte. However, in its current design, this system can only detect explosives contained or prepared in liquid.

\subsection{Explosives Detection Using a Fiber Bundle with a Functionalized End Face}

Dr. Walt's group developed a unique system capable of detecting vapor explosive substances [51]. Although their earlier detector used imaging fibers [52], in this review only their system based on large-core fiber is presented. As shown in Fig. 7(a), this system uses $1.52 \mathrm{~m}$ of fiber with an NA of 0.22 and a core diameter of $300 \mu \mathrm{m}$ to form a six-around-one bundle. The tip of each fiber is functionalized with different sensory material or material combinations, including AFPs from Dr. Swager's group at MIT. Each sensory material responds differently to vapor exposure so that the fluorescence response patterns are unique for each analyte. The instrument 


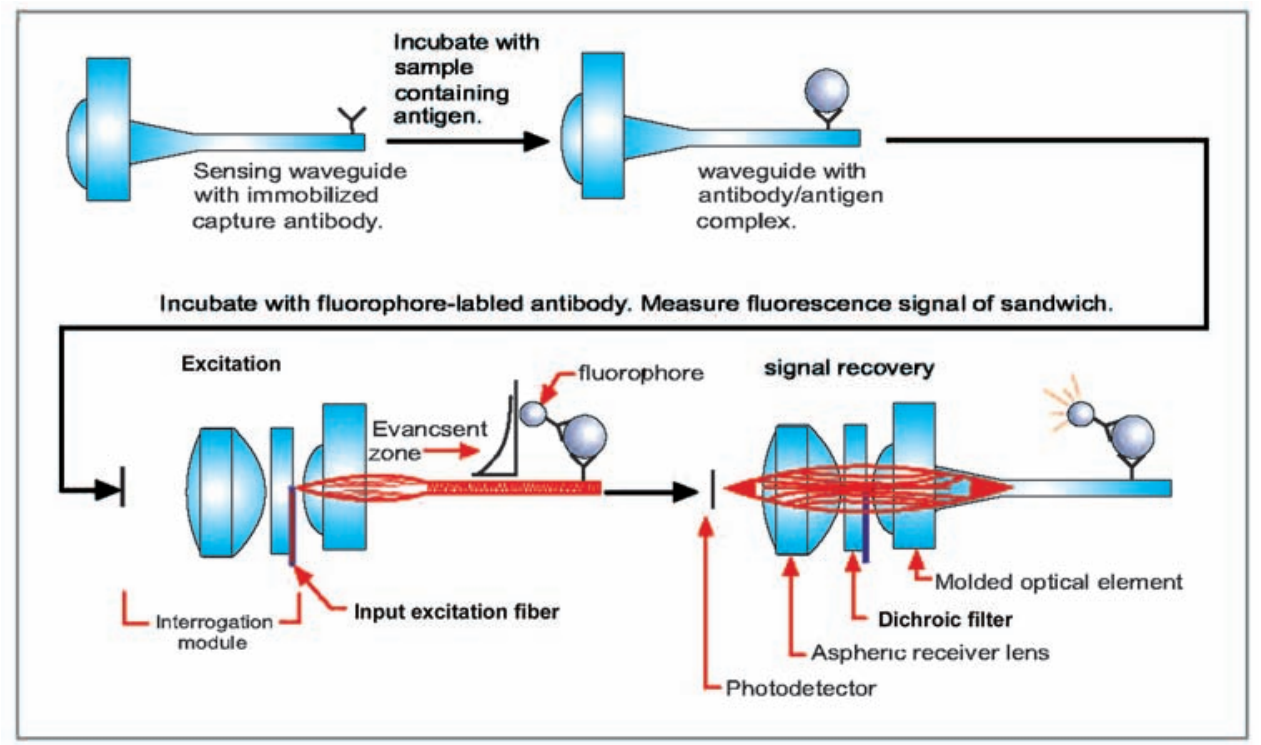

Fig. (6). Principle of operation of the antibody-antigen functionalized taper element adopted by Analyte 2000 and Raptor models.

is computationally "trained" to discriminate target response patterns from non-target patterns and background environments. Its optical system shown in Fig. 7(b) uses beam splitters F2 and F3 for excitation light and fluorescent signal delivery. Each fiber serves both to input excitation light from the LEDs and to transmit the fluorescent signal. A CCD camera is used to identify the responses from each individual sensory material. Fig. 7(c) shows the field-deployable system and Fig. 7(d) highlights the compact size of the sensing head (sniffer). This system has a capability of blind detection of 2,4-dinitrotoluene (2,4-DNT) to the level of $120 \mathrm{ppb}$.

\subsection{Explosives Detection Using Long Fiber with Func- tionalized Cladding}

Unlike the EW-based fiber-optic sensor system in (Fig. 6) where only very short fiber waveguides are used, the EWbased fiber-optic sensor system in (Fig. 8), also capable of explosive vapor detection, was built with $200-\mu \mathrm{m}$-core multimode fiber which may be as long as several meters for sensing, excitation and signal transmission purposes [53]. The middle segment of the fiber is decladded, coiled and coated with sensory materials based on triphenylene-ketals [54]. These receptors bind TNT molecules through charge transfer, causing attenuation of the transmitted light and a color change from transparent to red. This system can be used for online and in-situ TNT detection down to the subppb level.

\subsection{Explosives Detection Using a Capillary Waveguide}

The Fido explosives detector product line was originally developed by Nomadics, Inc., which later became ICX Nomadics, Inc. under ICX Technologies, Inc. and now is owned by Flir Systems, Inc. [55]. Rather than using a flexible optical fiber, the Fido product uses a rigid glass capillary for signal transmission and AFP coating. However, this capillary tube actually functions as a hollow-core circular waveguide. Fig. 9(a) illustrates the operating principle of the Fido model. The AFP is coated onto the inner wall of the capillary tube via the spin-coating process. Excitation light is sent directly from an external source which is positioned perpendicular to the tube via a free-space optics arrangement. The advantage of this design is that the AFP film receives the maximum possible excitation light which in turn generates the maximum fluorescent signal level. Part of this signal, entering the glass via EW-field coupling, will propagate from the excited AFP site to the detector. Additional advantages of this design are that minimal stray excitation light arrives at the detector and there is no need for extra housing to hold and evacuate the explosive vapor. Fig. 9(b) shows the principle of operation of the AFP adopted by the Fido model, which will be further discussed in the next section. Also shown are photographs of four Fido models. Fig. 9(c) is the Fido XT model used for screening people, vehicles or buildings with trace vapor detection ability, and it is robotmountable. Fig. 9(d) is the PaxPoint model for screening passengers, baggage, sealed containers and bottles. Fig 9(e) is the FastGate model which can be used to swipe any standard size ID or credit card to detect hidden explosives and Fig. 9(f) is the OnBoard system in which the handheld Fido model is mounted on a robot.

The Fido products can achieve a detection limit as low as one femtogram $\left(1 \times 10^{-15} \mathrm{~g}\right)$, a sensitivity 10 times higher than would be expected from the most sensitive IMS-based technology. Fido products were used by U.S. troops in Iraq and Afghanistan for the detection of IEDs.

\section{OVERVIEW OF AFP MATERIAL FOR THE DE- TECTION OF TRACE VAPOR EXPLOSIVES}

\subsection{Challenges for the Detection of Trace Vapor Explo- sives in Air}

Among the major advantages of vapor detection are that it is non-invasive and can be carried out inconspicuously, which makes it an ideal tool for counter-terrorist operations. Yet, a successful method for detecting vapor explosives must be able to measure traces of characteristic volatile compounds that evaporate from the explosive material, which is 

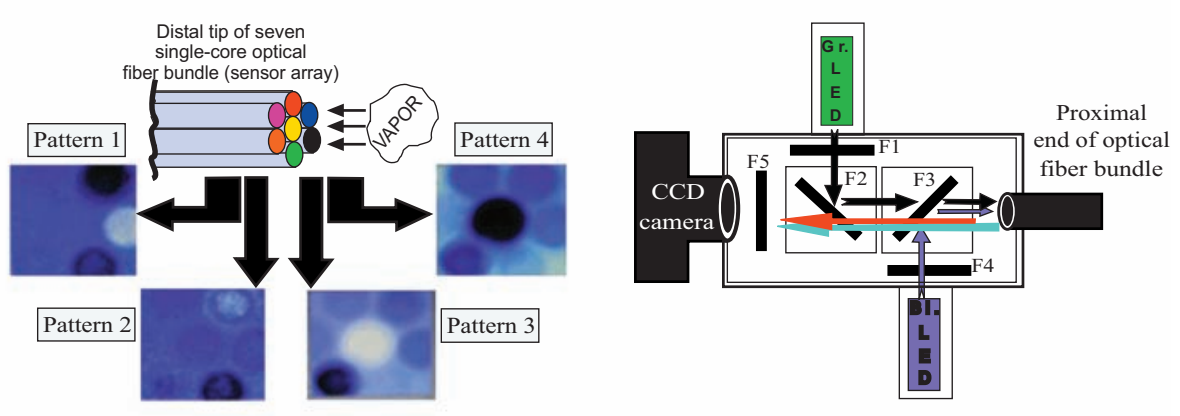

(a)

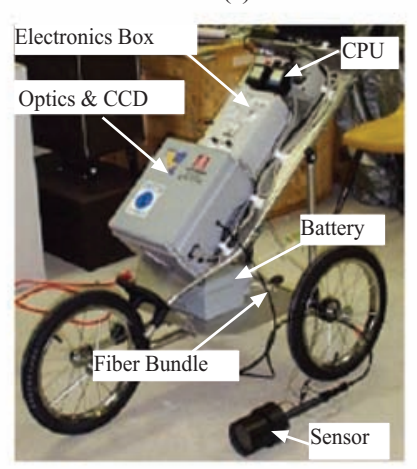

(b)

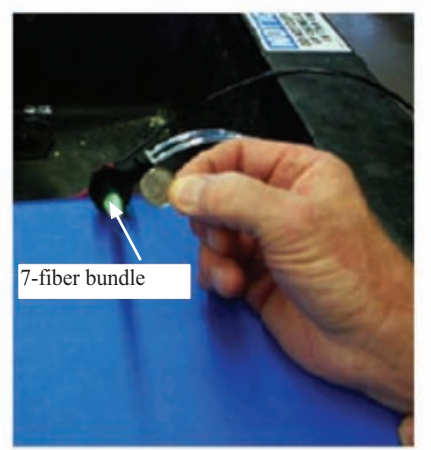

Fig. (7). Field-deployable system developed by Dr. Walt's group. (a) A fiber bundle with fiber tips coated with different sensory materials to generate unique patterns for explosives. (b) Operating principle of the system. (c) A system ready for field test. (d) Detector head smaller than a dime in size.

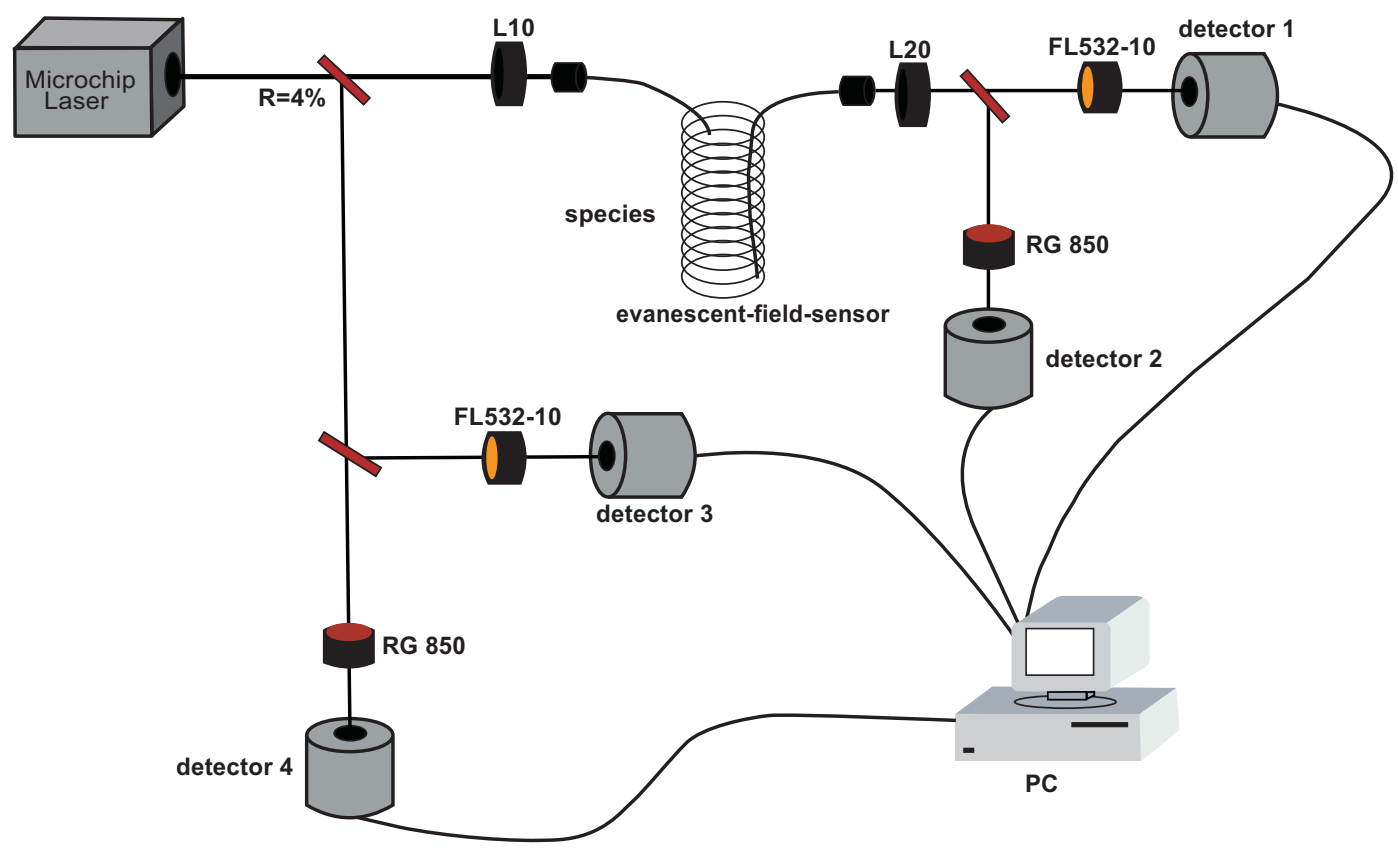

Fig. (8). An EW- based fiber-optic explosives detector employing large-core fiber for transmission of excitation and signal light as well as sensing purposes.

challenging due to their extremely low concentration (vapor pressure). This concentration relates to the volatility of the sample, which is characterized by the concentration of saturated vapors near the surface of an explosive. It has been reported in the literature that at a temperature of $25^{\circ} \mathrm{C}$ the vapor concentration is as follows: for nitroglycerin $-4.1 \times 10^{2}$ $\mathrm{ppb}$, for TNT - 7.7 parts per trillion (ppt), for PETN - $18 \mathrm{ppt}$ and for RDX - 6 ppt [46]. Yet these figures are not constant values but drop rapidly even at a short distance from the targeted surface, causing another drop of several orders of 
magnitude in the concentration in air. Additional factors such as low temperature, strong wind and well-chosen explosive packaging materials further worsen the conditions for detection. This explains why most vapor detection methods to date have proven capable of detecting trace vapor explosive materials on the surface but not in air $[11,56]$.

\subsection{The Detection of Trace Vapor Explosives with AFP}

AFP detects TNT and DNT explosives in the vapor phase with extremely high sensitivity, enabled by the tremendously amplified quenching effect produced by contact with vaporphase analytes. AFP consists of fluorescing chromophores linked together in polymer chains. In the absence of TNT, AFP fluoresces when excited. Binding of explosive molecules such as TNT to AFP quenches or "turns off" the fluorescence. As illustrated in Fig. 9(b), contrary to the traditional concept of fluorescence quenching, where binding of a single molecule of explosive quenches only the chromophore to which the explosive molecule is bound, with AFP, binding of a single molecule of explosive turns off the fluorescence of many chromophores, resulting in a dramatic amplification of the quenching. Analysis of the underlying mechanism shows that this fluorescent quenching is based on a photoinduced transfer of electrons from the excited polymer donor to the explosive acceptors. The unoccupied low-energy $\pi^{*}$ orbitals in nitroaromatic explosives accept an electron from the excited polymers, leading to non-bonding electrostatic interactions [57] between the electron-rich polymer and the electron-deficient DNT and TNT molecules.

The origin of AFP lies in research by Dr. Swager and coworkers at MIT. In 1998, they reported the detection of nitroaromatic explosives using fluorescent quenching of peniptycene-derived conjugated polymer [58]. The rigid peniptycene group prevents $\pi$-stacking of the polymer backbones, reduces interpolymer interactions and generates large enough cavities to allow diffusion of organic molecules into the film. More electron-rich polymers and larger cavities will produce a greater fluorescence response to nitroaromatic explosives. The AFP-based method has proven to be simple, sensitive and rapid. The quenching percentage depends on many factors, including the vapor pressure, the exergonicity of electron transfer, the strength of the binding interaction between the AFP and the explosives particles, and the ability of analytes to diffuse through polymer films.

\section{LOF DEVICES FOR THE DETECTION OF TRACE VAPOR TNT EXPLOSIVES}

This section onwards describes our research at the Université du Québec en Outaouais into an LOF device incorporating AFP film for the detection of trace vapor TNT explosive material. The principle of operation is based on fluorescence quenching of the film upon contact with the vapor of DNT, the precursor of TNT.

\subsection{LOF Architecture Accommodating Low-QY AFP}

An LOF-based platform wins over an analytical instrument in many respects, including fast response, portability, field-deployability, on-site detection capability and low cost. However, it cannot rival the analytical instrument in terms of the ability to send excitation light and to capture/deliver the signal light. While this problem might be alleviated by accumulating signal power over a long fiber as is the case with the EW- based detector shown in (Fig. 8), the use of a long fiber complicates packaging and will enlarge the detector. A better way for the LOF device to overcome these problems is by incorporating a highly sensitive sensory material such as AFP. A further advantage of the tiny LOF device is that it facilitates the connection with other parts of the system such as the pre-concentration unit. In recognition of these merits, we have devoted much of our research effort to the construction of LOF devices that can detect the fluorescent signals from highly sensitive AFPs. The main challenge being encountered arises from the fact that the AFPs from our collaborator have low QYs. The success of the LOF device thus depends on an optimized LOF architecture capable of collecting fluorescent light with the maximum possible efficiency from the AFP thin film, which is directly coated on the device surface.

Our optimized LOF architecture was inspired by existing fiber-optic probes based on large-core fibers for better light capturing ability [59-62]. This fact naturally led to our decision to use large-core fibers in our LOF device. Fig. (10) compares several typical fiber-optic sensing configurations using large-core fibers to capture the fluorescence power from AFP film coated on either the flat surface or the fibercore sidewall. Generally speaking, the one-fiber architecture has the best light-collection efficiency and the smallest sensing head but requires one or more additional elements such as a fiber coupler, a beam splitter, or a collimating-focusing assembly as well as their supporting fixtures, which poses technical issues for system construction and entails additional cost. Of course, if the system is well constructed and offers a longer service life for numerous repeated uses, this cost could be averaged out over the number of samples analysed. The fiber-optic biosensor systems illustrated in (Fig. 2 and Fig. 6) are good examples of this approach. Fig. 10(a) shows a one-fiber architecture using the front end of a largecore fiber to capture the light from film coated on a flat substrate, which is separated from the fiber. Additional fixtures are needed to hold the substrate and the light source. A substantial improvement of Fig. 10(a) is the structure in Fig. 10(b) that straightforwardly coats the AFP film on the sidewall of the core. The fluorescent light is captured via the EW tail. An additional fixture is needed for the light source, if free-space based. Fig. 10(c) shows another architecture using one fiber for both excitation/emission (Ex/Em) light transmission/collection. The sensing section is simplified but the problem of additional fixtures remains, as a beam splitter must be added. The tapered fiber end in Fig. 10(d) offers better Ex and Em intensity but shares the same problem as Fig. 10(c) in the need for additional fixtures. The greatly simplified architecture shown in Fig. 10(e) replaces the freespace optics with an additional excitation fiber (e-fiber) to deliver the Ex light to the film on the sensing fiber (s-fiber). Our LOF solution is shown in Fig. 10(f). By introducing a slanted end-face at the tip of the e-fiber to deflect Ex light delivery to the s-fiber sidewall, an elegant $1.6 \times 1.6 \times 0.8$ $\mathrm{mm}$ LOF architecture is created near the tips of the two fibers. This LOF device eliminates the problem of fixtures and additional optical components illustrated in Fig. 10(a-e) while offering sufficient Ex power, efficient fluorescent light collection and stray Ex light suppression. Both fibers are 


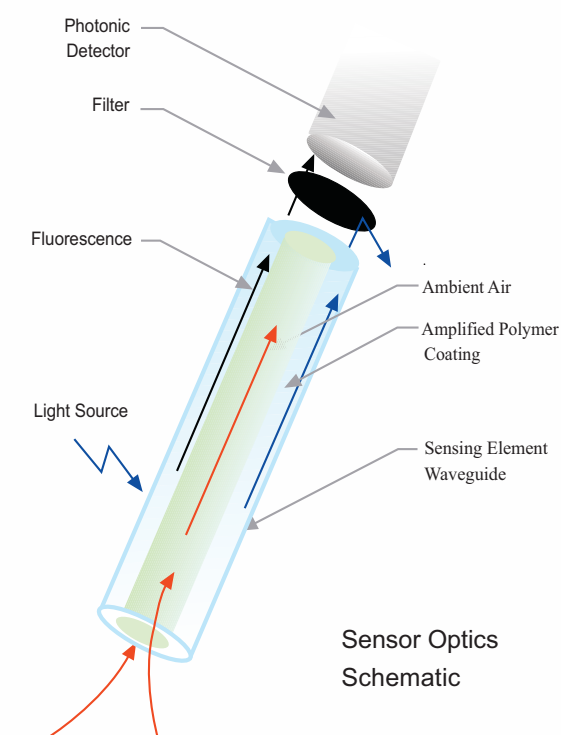

(a)

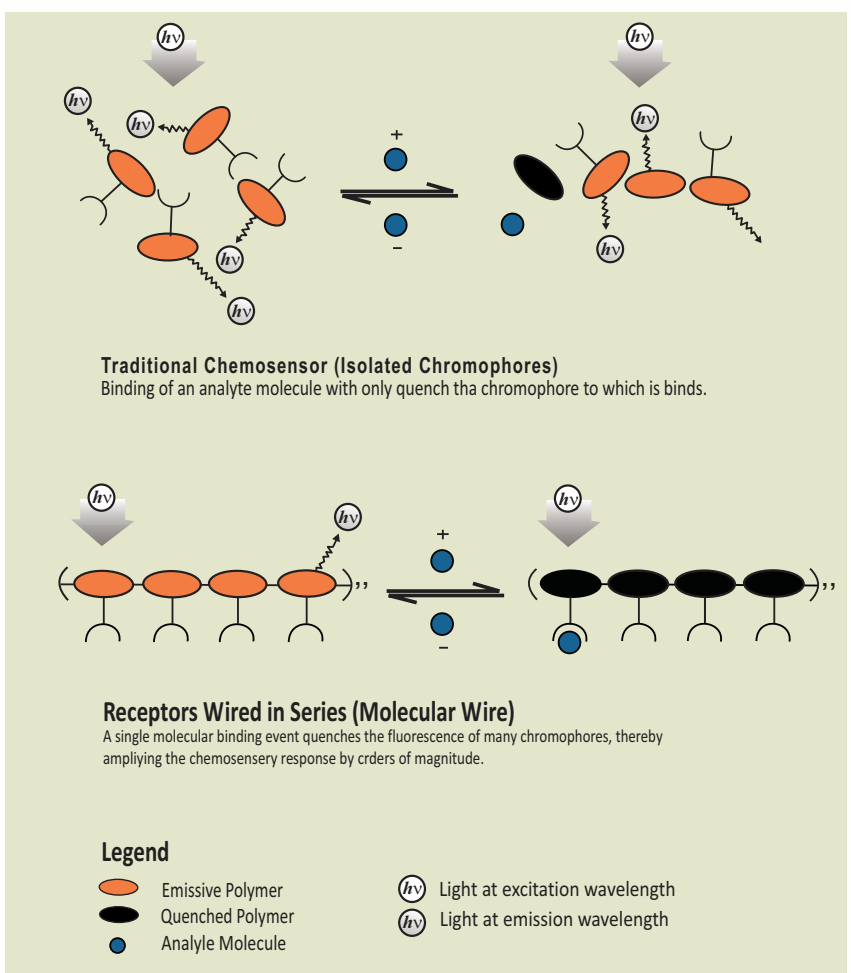

(b)

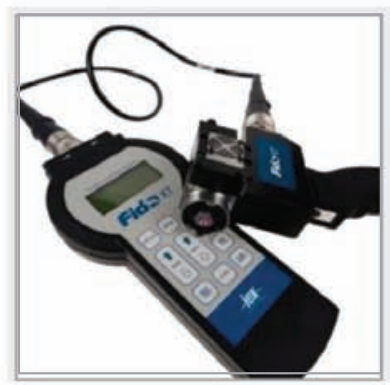

Fido $X T$

(c)

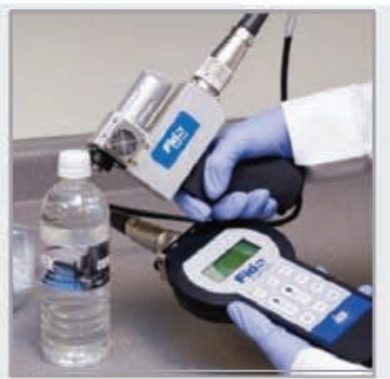

Fido PaxPoint

(d)

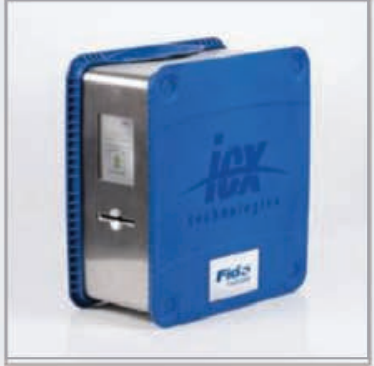

Fido FastGate

(e)

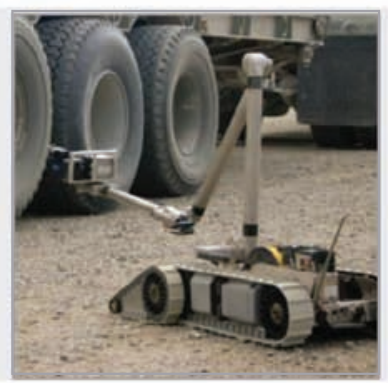

Fido OnBoard

(f)

Fig. (9). Fido explosives detector product series. (a) Principle of operation of the Fido product; (b) Principle of operation of AFP sensory material; (c) Photograph of Fido XT; (d) Photograph of Fido PaxPoint; (e) Photograpth of Fido FastGate; (f) Photograph of Fido OnBoard.

BFL37-800 from Thorlabs Inc. with $800 \mu \mathrm{m}$ core and NA of 0.37, which offer a large surface area for AFP film coating to build up the extremely weak fluorescent power from the low QY AFP film. Yet, even when a large-core fiber is adopted, the sensing section occupies mere a $1.6 \times 1.6 \times 0.8 \mathrm{~mm}$ cube, forming an elegant LOF device.

\subsection{The Synthesis and Properties of AFP Sensory Mate- rial}

Two series of fluorescence "turn-off" polymers, represented by polymers (PL) 4a, 4b and 5a, 5b, illustrated in (Fig. 11), were supplied by our collaborator, who synthesized them for the detection of NACs such as 2,4,6trinitrotoluene (TNT) [63]. These polymers were made by the Suzuki cross-coupling reaction of dibromide and bisboronic acid ester monomers. For PL $4 \mathrm{a}$ and $4 \mathrm{~b}$, the monomer was designed to have an electron-donating diphenylamine moiety, in order to probe any donor-acceptor interaction with the electron-accepting NACs, thus enhancing the detection sensitivity. PL 5a and 5b were synthesized by grouping the bulky cholesterol esters and long alkyl chains with the chosen monomers, thus creating a site-isolating group to prevent the interchain interaction and form a large free volume in the resulting polymers. Such polymers enhance fluorescence QY and help to trap analyte molecules.

\subsection{Uniform AFP Film Formation by Treating the Fiber with Adhesion Promoter}

The film on the sidewall of the s-fiber was deposited via dipping. To ensure the uniformity of the film, a proper pretreatment of the substrate with adhesion promoter is required to assist in bridging, or establishing a chemical bond 

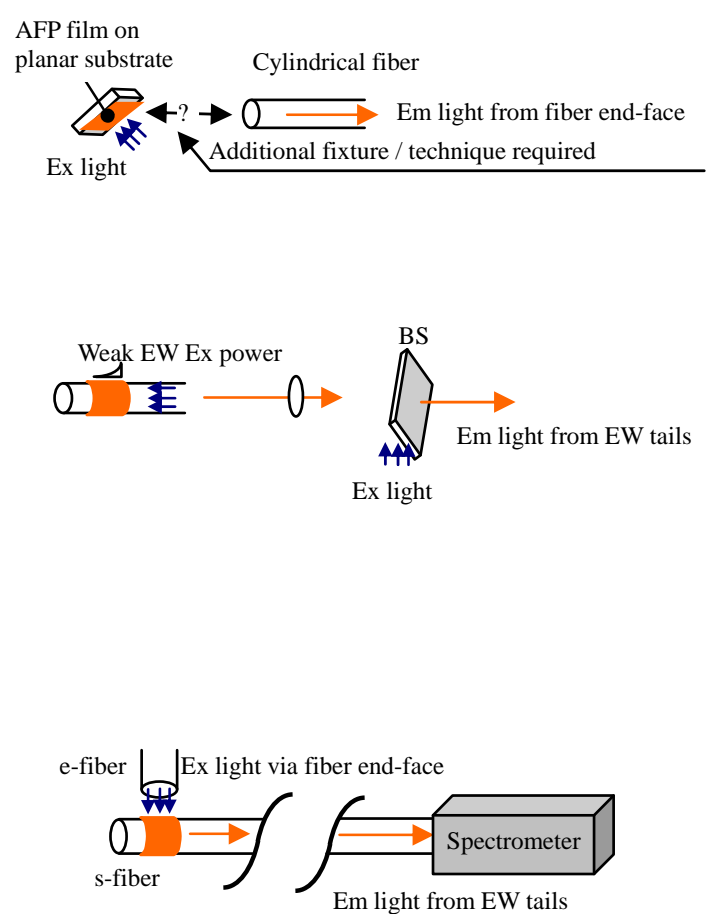
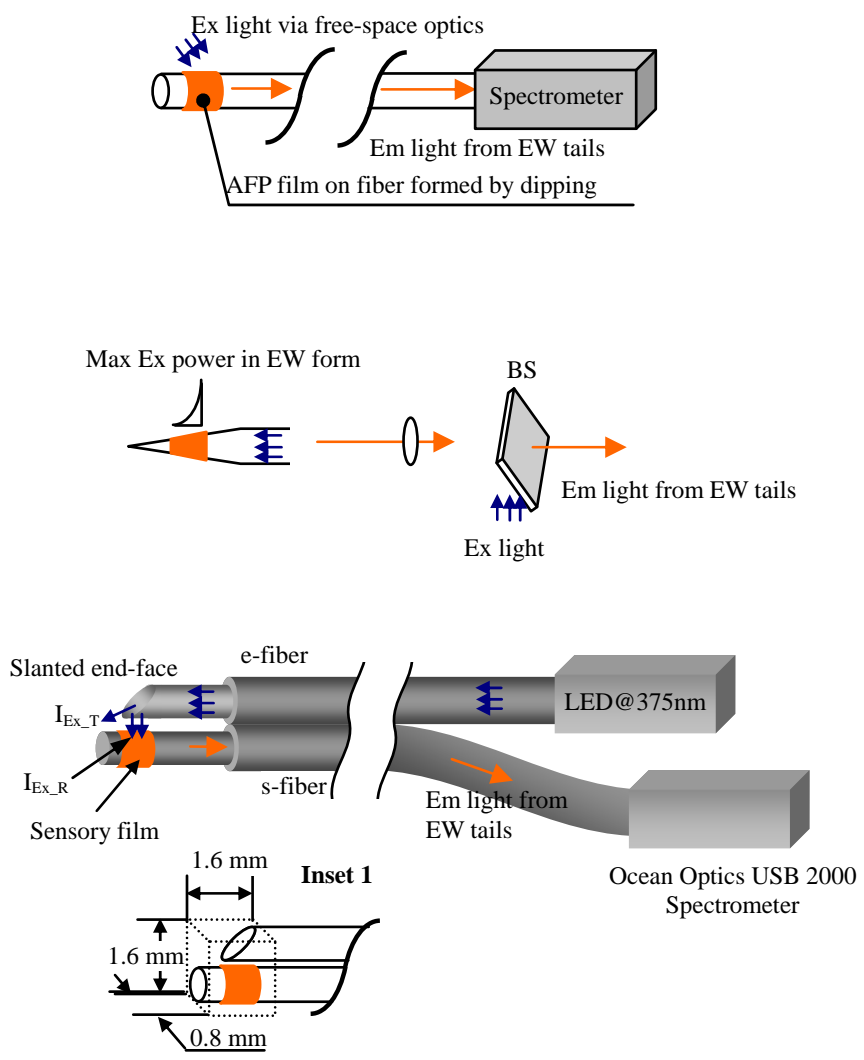

Fig. (10). Several typical fiber-optic sensing architectures for the detection of fluorescence of AFP film. (a) One-fiber architecture with the film on a flat substrate; (b) One-fiber architecture with the film on the sidewall of the core; (c) One-fiber architecture using one fiber for both Ex/Em light transmission/collection; (d) A tapered architecture offering better Ex and Em intensities than (c); (e) Architecture greatly simplified by adding an additional e-fiber for the Ex light delivery; (f) Our LOF architecture capable of efficiently capturing the weak fluorescent signal from low QY AFP film.

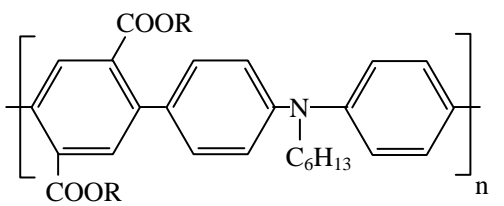

$4 \mathrm{a}$ or $4 \mathrm{~b}$

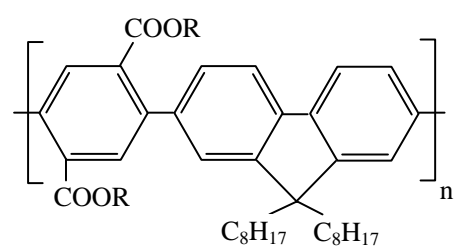

5 a or 5 b $\mathrm{a}=$

$\mathrm{b}=$<smiles>CC(C)CCCC(C)C1CCC2C3CC=C4CC(C)CCC4(C)C3CCC12C</smiles>

Fig. (11). Chemical structures of PL 5a, 5b, 4a and 4b for the detection of NACs such as TNT.

between the organic polymer and the inorganic substrate. We chose 3-aminopropyltrimethoxysilane, $\mathrm{H}_{2} \mathrm{~N}\left(\mathrm{CH}_{2}\right)_{3} \mathrm{Si}\left(\mathrm{OCH}_{3}\right)_{3}$ from Fluka as the adhesion promoter. The dipping process follows the sequence of promoter, water and TNT sensory polymer solution. Dipping into water provides the moisture needed for the silane's alkoxy groups to hydrolyze and react with the inorganic substrate, forming a bond covalently to the inorganic substrate such as the fiber core.

\subsection{Quenching Response of AFP Film on LOF vs. on Planar Substrate}

A series of experiments was performed to compare the quenching responses of APF film on this LOF device with that of films coated on planar glass substrates. A polymer solution (5.0 mg in $1.0 \mathrm{~mL}$ of chloroform) was spin-coated onto a glass substrate with a film thickness of $\sim 65 \mathrm{~nm}$. The polymer films on optic-fiber tips representing the LOF were dip-coated in chloroform solution $(1.5 \mathrm{mg} / \mathrm{mL})$ and air-dried. The film thickness was measured by atomic force microscope (AFM) to be $\sim 90 \mathrm{~nm}$.

TNT explosive vapor is simulated by its well-known precursor, 2,4-dinitrotoluene (DNT). The DNT powder is sealed in a bottle measuring $\phi 45 \times 50 \mathrm{~mm}$. The DNT vapor pressure created within the bottle is about $1.74 \times 10^{-4} \mathrm{mmHg}\left(25^{\circ} \mathrm{C}\right)$ [63]. The LOF-based system is shown in Fig. 10(f). The quenching effect can be easily monitored and recorded con- 
tinuously by keeping the tiny LOF device inside the bottle via a small $\phi 10 \mathrm{~mm}$ opening. The e- and s-fiber leads are connected to an LED with its central wavelength at $375 \mathrm{~nm}$ and a hand-held spectrometer (USB 2000 from Ocean Optics), respectively. To monitor the fluorescent quenching from the AFP on planar substrate, first the film-coated substrate is immersed in the bottle containing the DNT vapor for a specific period of time. Second, it is removed from the bottle and immediately inserted into the lab-based analyticallevel spectrometer for spectrum recording. By repeating the two steps, the quenching percentages versus different exposure times are obtained.

The experimental results for all four polymer materials, PL 4a, 4b, 5a and 5b, are plotted in (Fig. 12). For a planar substrate, the first 50 seconds is inaccessible since this is the setup time needed to manually transfer the sample substrate from the DNT exposure site to the lab-based spectrometer before data acquisition. Notwithstanding this setup time, the trends of curves in (Fig. 12) clearly show that for all four materials, the LOF device demonstrated far superior performance in responding to the presence of the DNT vapor.
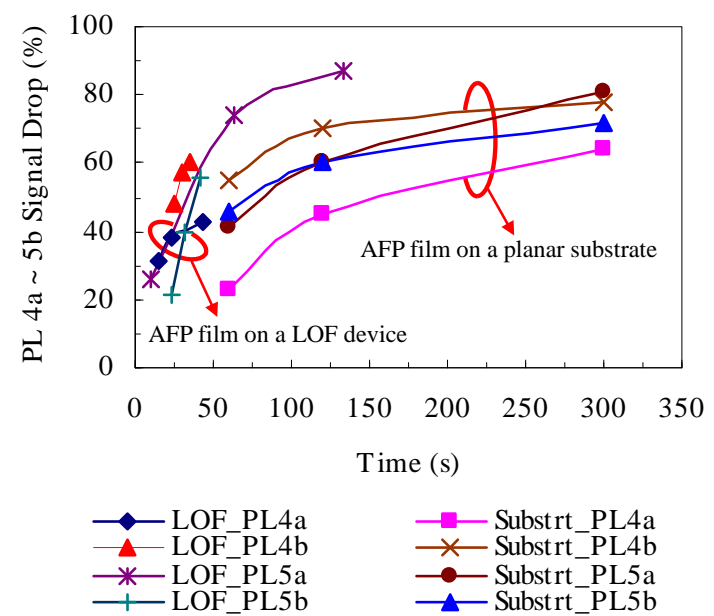

Fig. (12). Evaluation of fluorescent quenching for LOF devices and planar substrates coated with AFP films. Curves include the results from all four polymers, PL 4a, 4b, 5a and 5b. LOF devices offered much faster responses to the DNT vapor.

\section{LOF DEVICE FOR THE DETECTION OF VAPOR TNT EXPLOSIVES: FURTHER IMPROVEMENT}

Fig. (13) shows another LOF device, which is a short fiber taper with AFP film. The device takes a tiny space of $\phi 0.4 \mathrm{~mm} \times 1 \mathrm{~mm}$. Quick and reliable connections are ensured by SMA connectors. The end face of the untapered end of the sensing section overlaps two fibers of the bifurcated fiber bundle with core/cladding diameter of $0.2 / 0.23 \mathrm{~mm}$ to simultaneously receive the excitation light and send the captured signal. Stand-off or remote detection is enabled by prolonging the two leads of the bifurcated fiber bundle to arbitrary lengths.

Fig. (14) gives the setup of a cost-effective system we built for fast, easy and effective evaluation of this LOF device. The same DNT vapor pressure of $1.74 \times 10^{-4} \mathrm{mmHg}$ $\left(25^{\circ} \mathrm{C}\right)$ described in the previous section is established in the sealed bottle. Fig. (15) indicates that this LOF device offers a dramatic fluorescence turn-off of $31 \%$ within 12 seconds upon contacting the DNT vapor. A signal drop of $47 \%$ is observed after 24 seconds. The further turn-offs with longer time spans are clearly illustrated by Fig. 15(b) and (c), which show that after 40 seconds the drop in signal levels off.

\section{INVESTIGATION OF SEVERAL CRITICAL IS- SUES RELATED TO AFP FILM}

\subsection{Effect of Polymer Concentrations on the Quenching Performance of the LOF Device}

The thickness of the film on the fiber sidewall is a decisive factor in the performance of the LOF device. The thinner the film, the more free volumes or cavities contained in the polymer are available to participate in DNT molecule trapping, enabling a fast response to the presence of the explosive. Besides, the very thin film tends to fully open these cavities to air, which makes it easier to clean out the trapped analytes with purge gas after measurements are taken, leaving the device ready for its next use. In contrast, a thick film will offer a stronger initial fluorescent signal. However, the greater thickness means that some cavities may be deeper, while others remain either partially or completely hidden under the surface layer of the film. As a result, the analyte

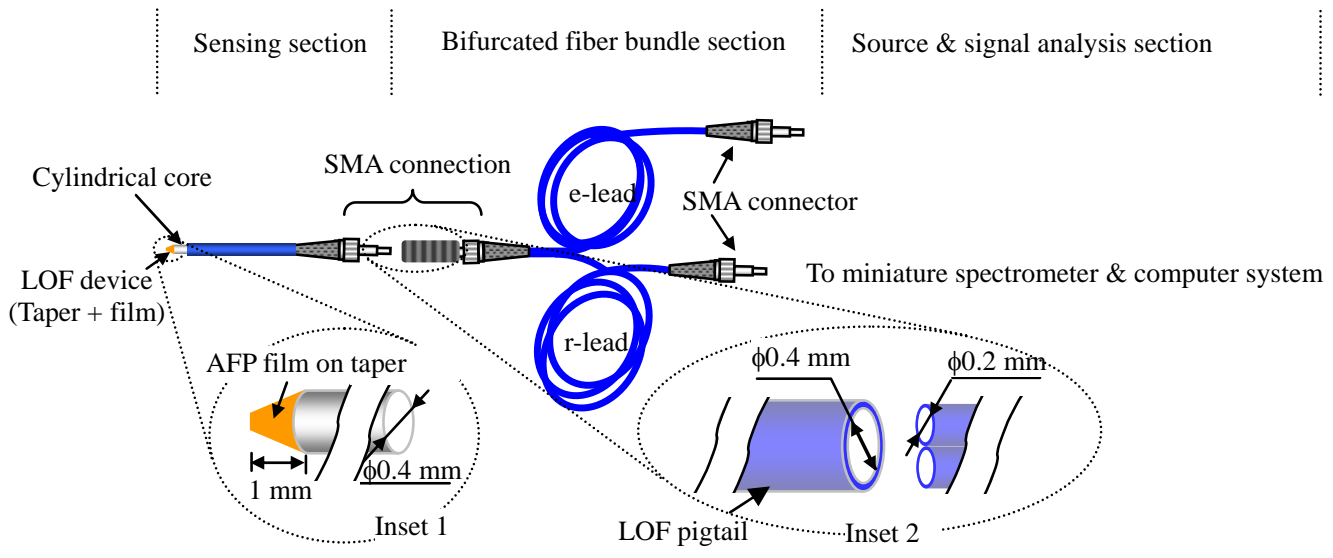

Fig. (13). Architecture of LOF device built on a short fiber taper and its connection with the bifurcated fiber bundle. Inset $1: \phi 0.4 \mathrm{~mm} \times 1$ mm taper coated with AFP film constitutes the LOF device; Inset 2: Untapered end of the sensing section overlaps two fibers of the bifurcated fiber bundle. 

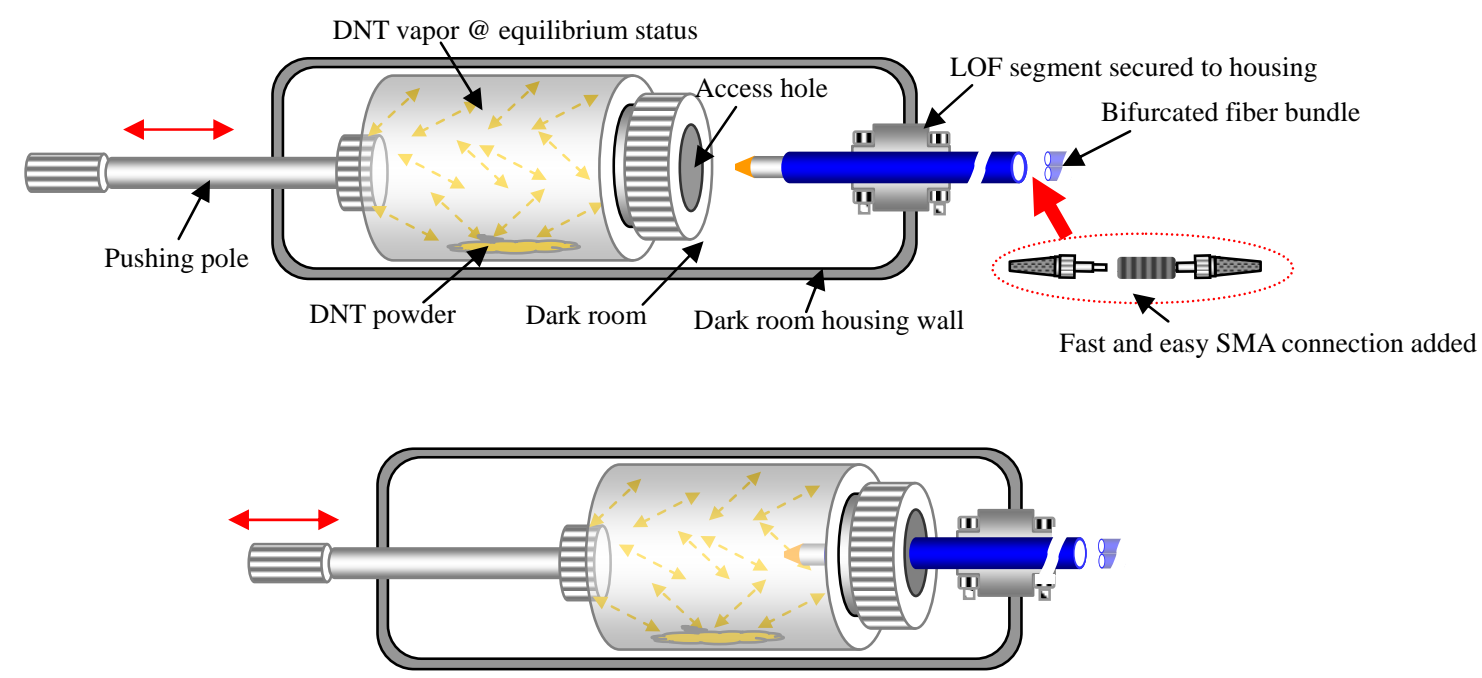

Fig. (14). Setup for the detection of DNT vapor. (a) LOF before DNT vapor exposure; (b) LOF during DNT vapor exposure.
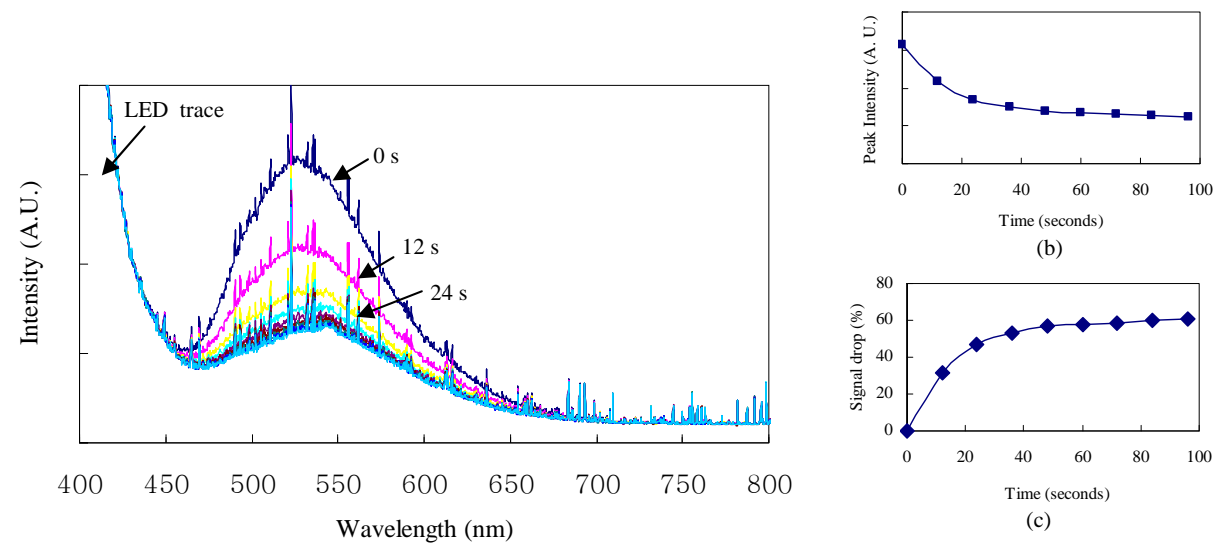

(b)

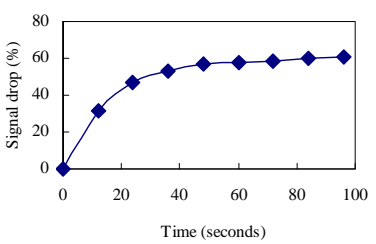

(c)

(a)

Fig. (15). Experimental results of the fluorescence turn-off of the improved LOF device. (a) Fluorescence spectra showing the turn-offs when the fluorescence spectrum is captured every 12 seconds. A dramatic 31\% fluorescence turn-off occurs in the first 12 seconds. (b) Fluorescence intensity vs time, showing the fast turn-off in the first 40 seconds which levels off afterwards. (c) Fluorescence turn-off in terms of the percentage of fluorescence intensity drop.

trapping process is slow, as is the quenching process. Another obvious side effect is the difficulty of removing analytes trapped farther down in the film.

Unlike the spin-coating process that can control film thickness by regulating the coater spinning speed, the dipping process relies on varying the concentration of the polymer solution to optimize the film thickness. For PL 4a, we prepared four solutions with mass concentrations of $0.02 \%$, $0.03 \%, 0.07 \%$ and $0.13 \%$. Responses of the LOFs with thin films formed by these solutions are illustrated in (Fig. 16). The fluorescent intensities are recorded after 10-, 20- and 30second exposures to the DNT vapor.

Fig. (16) indicates that while a lower concentration leads to a thinner film and thus a faster response, further reduction of the concentration is limited by the fluorescent signal detecting system. This limitation becomes more obvious when the miniaturized detecting system with limited sensitivity is used to detect weak fluorescence. For example, the USB
2000 spectrometer in our system has a small footprint but requires a relatively long integration time to boost the weak fluorescent signal from the low QY AFP film to an acceptable level. Furthermore, we found that the quenching response may be compromised by the significantly broadened stray excitation light resulting from the long integration time, which is evidenced by Fig. 15(a). We resolved this issue by seeking a trade-off concentration over experiments and found the concentration of $0.03 \%$ demands a shorter integration time of $5 \mathrm{~s}$ but significantly improves signal quality.

\subsection{Effect of Ambient Light on the Fluorescent Quench- ing Performance}

Ambient light exposure of the AFP film can severely degrade the film in terms of quenching percentage. This effect fortunately can be mitigated as the LOF device has to be sealed in a hermetic chamber such as the adaptor module described in the next section to receive concentrated vapor 
from the pre-concentrator during operation. Fig. (17) shows how the ambient light affects the quenching response of the film, with exposure reducing the quenching percentage.

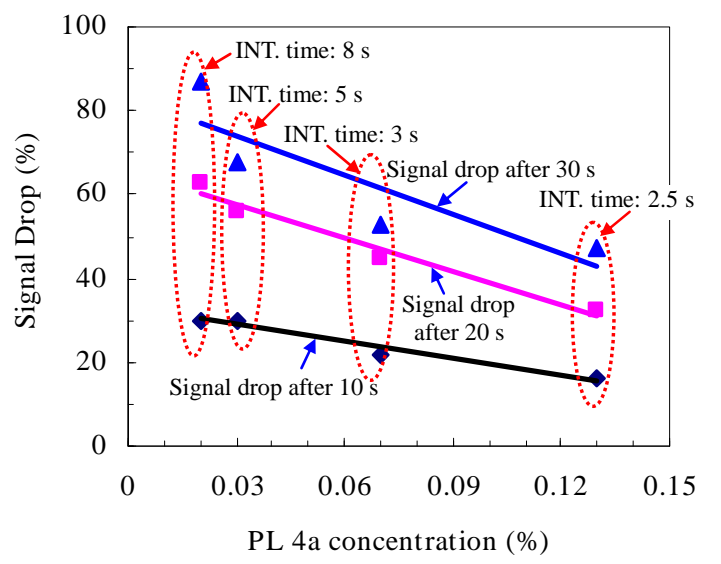

Fig. (16). Fluorescent quenching responses of the LOF to DNT vapor with different thin film thicknesses adjusted by dipping into the polymer solutions with the following concentrations: $0.02 \%$, $0.03 \%, 0.07 \%$ and $0.13 \%$ with chloroform as the solvent. INT. time: integration time.

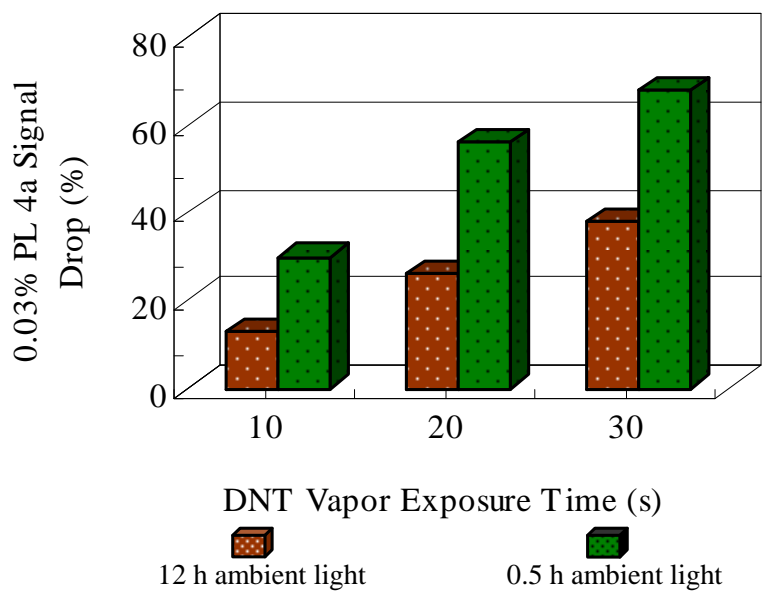

Fig. (17). Ambient light effect on the fluorescent quenching percentage for film formed by $0.03 \%$ PL 4 a polymer concentration.

\subsection{Storage-Life Evaluation of the Polymer Sensory Film}

The oxygen contained in the air also degrades AFP film. We examined the long-term storage life of these polymers when subject to oxygen alone. Fig. (18) gives the results for the film made from $0.03 \%$ PL 4a solution for a 24-day time span. Degradation of the film in terms of reduced quenching percentages indeed occurs over this period but the process is more gradual than with the ambient light effect. A much longer life span is expected if the space containing the LOF device is filled with nitrogen gas.

\section{OVERVIEW OF THE PRE-CONCENTRATION TECHNIQUE}

The tiny LOF device is fully compatible with the explosive vapor pre-concentrator, a critical unit in the instrumenta- tion system to minimize the probability of both missed detection and false alarms and to achieve on-line detection. The connection between the LOF device and the main body of the pre-concentrator, however, has its specific requirements. Fig. (19) shows the adaptor module we propose to properly establish this connection. The module has a small internal chamber and contains the following fundamental elements: an inlet for concentrated explosive vapor; an inlet to bring in purge gas to clean the detector surface after measurement; and an outlet to get rid of the waste. The design of this module must take into consideration the concentration of the sample, which is the trace vapor explosive in air. In this case, a ppt level of concentration is common, making the preconcentrator a mandatory addition. This low concentration also means that the number of collectable vapor molecules is limited and that the space within the adaptor module must be minuscule to maintain the highest possible concentration for detection. The LOF device is obviously the perfect candidate to fit into the tiny chamber of the adaptor module.

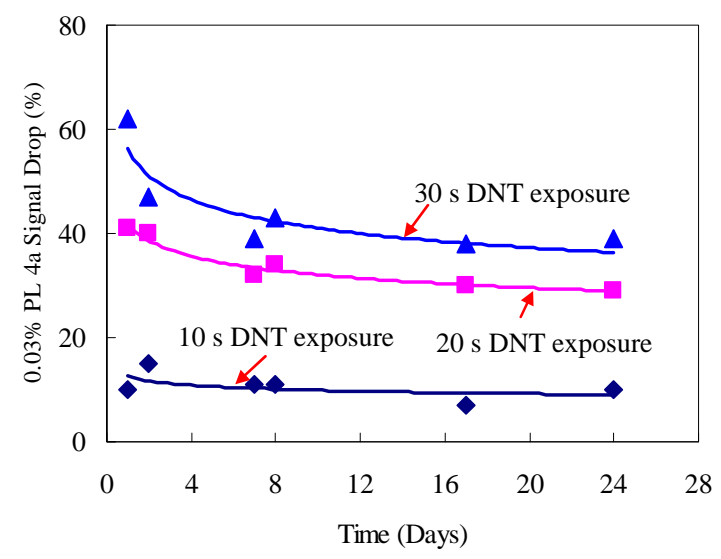

Fig. (18). Evaluation of storage life of PL 4a film over 24 days' time.

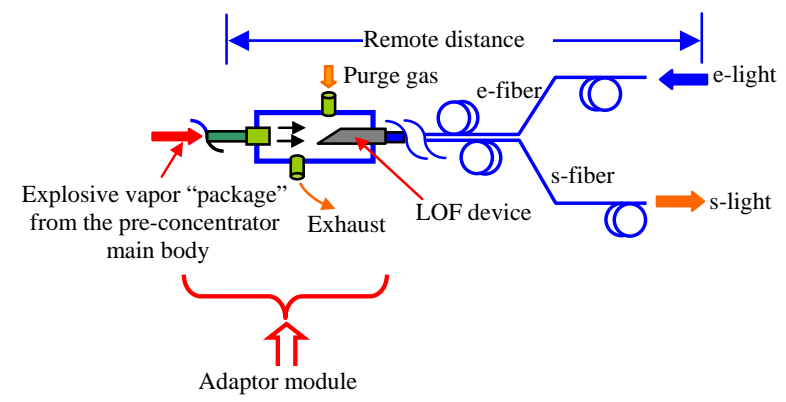

Fig. (19). Example showing an LOF device in the adaptor module.

The importance of including a pre-concentration unit for the LOF device is further justified by the following facts. Despite the high sensitivity of AFP, it is impossible to use a tiny LOF device to directly detect vapors that are sparsely distributed in a large volume of air. Adding to the problem of low concentration is another property of explosive vapor: its stickiness. Upon contact, explosive vapors stick to any object such as metal surfaces or the inner wall of a tube [64]. The pre-concentrator overcomes these problems by collecting a 
large volume of surrounding air, filtering or trapping the target particles in a miniaturized space and finally delivering them to the detecting zone. The heated air flow plays an important role in this process by effectively freeing any vapor molecules that are stuck. The Fido hand-held unit illustrated in (Fig. 9) achieves its femtogram detection limit by adopting a sophisticated pre-concentration and GC technique, which is well described by the patent of Nomadics Inc. [65]. The following components or factors are included or considered in their Fido model:

- A vacuum pump assists in drawing in a large volume of surrounding air with an optimum air-flow rate through a flow meter;

- An adsorption/desorption zone traps/screens/releases the explosive vapor on the internal wall for maximum performance;

- Multiple analytes are further separated by establishing a GC zone;

- Several temperature controllers in different zones manage heating/cooling cycles and the speed of the change from cooling to heating;

- Unwanted vapor-form quenchers other than the target analyte are removed since they may trigger a false alarm;

- A highly concentrated vapor package in the form of a "pulse" is created during the adsorption/desorption process;

- Ambient light is rejected at the location of inlet;

- An equilibrium zone matches the temperatures of the vapor and the AFP to eliminate fluctuation of the signal caused by temperature variations.

The consideration of these factors is fundamental in the development of a pre-concentration system for detecting trace vapor from explosives.

\section{CONCLUSIONS}

We have summarized recent progress in explosives detection with the emphasis on an approach based on fiberoptic sensors, particularly the LOF concept we first proposed in 2010 [1] and the LOF devices we subsequently demonstrated. Special attention is given to the pre-concentration technique that is crucial for the detection of trace vapor explosive substances in air. We have also explored the extremely active recent research on LIBS and instruments based on Raman scattering, highlighting their rapid evolution from benchtop instruments to portable units with stand-off or remote detection ability. These advances are driven by their ability to identify explosives instantly and to detect virtually any substance, plus the fact that their operation is nondestructive and requires no sample preparation.

The technical challenges in detecting explosives dictate the necessary coexistence of numerous detection means. Some methods are capable of detecting virtually any chemical species, or are all-inclusive, but this does not mean they are free of shortcomings. Both the cost and size of detection systems, and the need for experienced operators to cope with noise-rich spectra captured from samples with complex surrounding interferences set limits for their mass deployment.
Specifically, operators must be highly qualified in order to minimize false alarms, making operator costs one of the highest costs in such systems. In contrast, methods that target one or a small number of explosive species, i.e., fewinclusive methods, are often cost-effective, portable, robust and highly selective and require little training, so that they can easily be applied in a variety of environments. The fact that the explosives of greatest interest include only five species, namely TNT, RDX, HMX, PETN and TATP (Table 1), lends weight to the choice of few-inclusive methods. However, the more realistic way to cope with explosives detection in a complex environment is to apply both all-inclusive and few-inclusive methods instead of adopting only one technique. This allows the different detecting means to complement each other as, for example, Raman spectroscopy complements LIBS, and ensures that explosive substances can be rapidly identified with the lowest possible false alarm rate. Fiber-optic-sensor- or LOF-based systems usually deal with one or a few explosive species and can be considered as a verification tool to support all-inclusive methods. Of course, advances in technology have significantly downsized all-inclusive instruments such as those based on LIBS, but the challenge of how to properly process the captured spectra remains, leading to the same old demand for highly experienced operators. In the final analysis, it is wise to look to the combination of complementary techniques as the best guarantee of high-confidence detection.

Finally, we successfully demonstrated that our LOF devices coated with AFP films can detect low-pressure DNT vapor trapped in a bottle. This proves that our LOF devices can respond rapidly to the concentrated vapor in the adaptor module described in (Fig. 19). The next stage is thus how to incorporate a pre-concentration unit and an adaptor module with the LOF device and how to synchronize their operations to achieve true on-online detection of trace vapor explosive materials. This demands coordinated research by a multidisciplinary team with members from optics, polymer chemistry and analytical chemistry, which is ongoing in our research center at the Université du Québec en Outaouais.

\section{CONFLICT OF INTEREST}

The authors confirm that this article content has no conflicts of interest.

\section{ACKNOWLEDGEMENT}

The authors gratefully acknowledge support for this work from the Natural Sciences and Engineering Research Council of Canada and the Canada Research Chairs Program.

\section{REFERENCES}

[1] Ma J, Kos A, Bock WJ, et al. Lab-on-a-Fiber: Building a fiberoptic sensing platform for low-cost and high-performance trace vapor TNT detection. 2010 4th European Workshop on Optical Fiber Sensors. In: Santos J L, Culshaw B, López-Higuera JM, MacPherson WN (Eds). Proc SPIE 2010; 7653: 76531E-76531E-4.

[2] Ma J, Kos A, Bock WJ, et al. TNT vapor detection based on a Labon-a-fiber: Achieving a millimeter-scale sensing element on fiber. IEEE Sens J 2012; 12: 13-7.

[3] Ma J, Kos A, Bock WJ, et al. Lab-on-a-fiber device for trace vapor TNT explosive detection: Comprehensive performance evaluation. J Lightwave Technol 2012; 30: 1127-1133. Available from: http://www.opticsinfobase.org/jlt/abstract.cfm?URI=jlt-30-8-1127 
[4] http://www.oceanoptics.com/applications/samplesetups_libs.asp. September 4, 2013.

[5] Christian B, Konrad H, Dirk S, et al. All-fiber-coupled laserinduced breakdown spectroscopy sensor for hazardous materials analysis. Spectrochim Acta Part B: Atomic Spectroscopy 2007; 62(12): 1519-27.

[6] Shriver-Lake LC, Brian LD, Ligler FS. On-site detection of TNT with a portable fiber optic biosensor. Environ Sci Technol 1997; 31(3): 837-41.

[7] Bakaltcheva IB, Ligler FS, Patterson CH, Shriver-Lake LC. Multianalyte explosive detection using a fiber optic biosensor. Anal Chim Acta 1999; 399(1-2): 13-20.

[8] Department of the Treasury, Bureau of Alcohol, Tobacco and Firearms. Commerce in explosives: List of explosive materials. Federal Register /Notices, Apr 26, 2002; 67(81): 20864-20866.

[9] Leahy-Hoppa MR, Fitch MJ, Osiander R. Terahertz spectroscopy techniques for explosives detection. Anal Bioanal Chem 2009; 395: 247-57.

[10] Ewing RG, Atinson DA, Eiceman GA, Ewing G J. A critical review of ion mobility spectrometry for the detection of explosives and explosive related compounds. Talanta 2001; 54(3): 515-29.

[11] Caygill JS, Frank D, Seamus PJH. Current trends in explosive detection techniques. Talanta 2012; 88: 14-29.

[12] Yinon J., Ed. Counterterrorist Detection Techniques of Explosives, Vol. 1. Elsevier, Oxford: UK 2007.

[13] Song Y, Cooks RG. Atmospheric pressure ion/molecule reactions for the selective detection of nitroaromatic explosives using acetonitrile and air as reagents. Rapid Commun Mass Spectrom 2006; 20: 3130-8.

[14] Takada Y, Nagano H, Suga M, et al. Detection of military explosives by atmospheric pressure chemical ionization mass spectrometry with counter-flow introduction. Propell Explos Pyrotech 2002; 27: 224-8.

[15] Mulligan CC, Talaty N, Cooks RG. Desorption electrospray ionization with a portable mass spectrometer. Chem Commun 2006; 1709-1711.

[16] Woolard DL, Brown ER, Pepper M, Kemp M. Terahertz frequency sensing and imaging: A time of reckoning future applications? Proc IEEE 2005; 93(10): 1722-43.

[17] Liu H, Zhong H, Karpowicz N, Chen Y, Zhang X. Terahertz spectroscopy and imaging for defense and security applications. Proc IEEE 2007; 95(8): 1514-27.

[18] Federici JF, Schulkin B, Huang F, Gary D, Barat R, Oliveira F, Zimdars D. THz imaging and sensing for security applicationsexplosives, weapons and drugs. Semicond Sci Technol 2005; 20: S266-S280.

[19] Liu H, Chen Y, Bastiaans GJ, Zhang X. Detection and identification of explosive RDX by THz diffuse reflection spectroscopy. Opt Express 2006; 14: 415-23.

[20] Chen J, Chen Y, Zhao H, Bastiaans GJ, Zhang X. Absorption coefficients of selected explosives and related compounds in the range of 0.1-2.8 THz. Opt Express 2007; 15: 12060-7.

[21] Primera-Pedrozo OM, Soto-Feliciano YM, Pacheco-Londono LC, Hernandez-Rivera SP. Using reflection absorption infrared spectroscopy with fiber coupled grazing angle probe / FTIR. Sens Imaging 2009; 10(1): 1-13.

[22] Ramos C, Dagdigian PJ. Effect of photochemistry on molecular detection by cavity ringdown spectroscopy: Case study of an explosive-related compound. Appl Opt 2007; 46: 6526-32.

[23] Pacheco-Londono LC, Ortiz-Rivera W, Primera-Pederozo OM, Hernandez-Rivera SP. Vibrational spectroscopy stand-off detection of explosives. Anal Bioanal Chem 2009; 395: 323-35.

[24] Bernasconi CF. Kinetic and spectral study of some reactions of 2,4,6-trinitrotoluene in basic solution. I. deprotonation and Janovsky complex formation. J Org Chem 1971; 36: 1671-9.

[25] Gao D, Wang Z, Liu B, Ni L, Wu M, Zhang Z. Fluorescence quenching at the surface of silica nanoparticles toward ultrasensitive detection of TNT. Anal Chem 2008; 80: 8545-53.

[26] Rabenecker P, Pinkwart K. A look behind electrochemical detection of explosives. Propell Explos Pyrotech 2009; 34: 274-279.

[27] Wang J. Electrochemical sensing of explosives. Electroanalysis 2007; 19: 415-23.

[28] Agüí L, Vega-Montenegro D, Yáñez-Sedeño P, Pingarrón JM. Rapid voltammetric determination of nitroaromatic explosives at electrochemically activated carbon-fibre electrodes. Anal Bioanal Chem 2005; 382: 381-7.
[29] Shi G, Qu Y, Zhai Y, et al. \{MSU/PDDA\}n LBL assembled modified sensor for electrochemical detection of ultratrace explosive nitroaromatic compounds. Electrochem Commun 2007; 9: 17191724.

[30] Fu X, Chen X, Wang J, Liu J, Huang X. Amino functionalized mesoporous silica microspheres with perpendicularly aligned mesopore channels for electrochemical detection of trace 2,4,6trinitrotoluene. Electrochim Acta 2010; 56: 102-7.

[31] Lu D, Cagan A, Munoz RAA, Tangkuaram T, Wang J. Highly sensitive electrochemical detection of trace liquid peroxide explosives at a Prussian-blue 'artificial-peroxidase' modified electrode. Analyst 2006; 131: 1279-81.

[32] Parajuli S, Miao W. Sensitive determination of hexamethylene triperoxide diamine explosives using electrogenerated chemiluminescence enhanced by silver nitrate. Anal Chem 2009; 819(13): 5267-72.

[33] Wang J, Pumera M. Microchip flow-injection analysis of trace 2,4,6-trinitrotoluene (TNT) using mercury-amalgam electrochemical detector. Talanta 2006; 69(4): 984-987.

[34] Chuang M, Windmiller JR, Santhosh P, Ramirez GV, Galik M, Chou T, Wang J. Textile-based electrochemical sensing: Effect of fabric substrate and detection of nitroaromatic explosives. Electroanalysis 2010; 22: 2511-8.

[35] Nagatomo K, Kawaguchi T, Miura N, Toko K, Matsumoto K. Development of a sensitive surface plasmon resonance immunosensor for detection of 2,4-dinitrotoluene with a novel oligo (ethylene glycol)-based sensor surface. Talanta 2009; 79: 1142-8.

[36] Singh P, Onodera T, Mizuta Y, Matsumoto K, Miura N, Toko K. Dendrimer modified biochip for detection of 2,4,6 trinitrotoluene on SPR immunosensor: Fabrication and advantages. Sens Actuators B: Chem 2009; 137: 403-409.

[37] Meaney MS, McGuffin VL. Luminescence-based methods for sensing and detection of explosives. Anal Bioanal Chem 2008; 391: 2557-76.

[38] Bunte G., Hurttlen J, Pontius H, Hartlieb K, Krause H. Gas phase detection of explosives such as 2, 4, 6-trinitrotoluene by molecularly imprinted polymers. Anal Chim Acta 2007; 591: 49-56.

[39] Wang J, Hocevar SB, Ogorevc B. Carbon nanotube-modified glassy carbon electrode for adsorptive stripping voltammetric detection of ultratrace levels of 2,4,6-trinitrotoluene. Electrochem Commun 2004; 6(2): 176-9.

[40] Lubczyk D, Siering C, Lorgen J, Shifrina ZB, Mullen K, Waldvogel SR. Simple and sensitive online detection of triacetone triperoxide explosive. Sens Actuators B: Chem 2010; 143: 561-566.

[41] Nina LL, Roger CW, Samuel MC, Ann MO, Seth DH, Horton EN, James EB. Calibrating the ChemCam laser-induced breakdown spectroscopy instrument for carbonate minerals on Mars. Appl Opt 2010; 49: C211-C217.

[42] Jennifer LG, Frank CDL, Chase AM, Andrzej WM. Laser-induced breakdown spectroscopy for detection of explosives residues: A review of recent advances, challenges, and future prospects. Anal Bioanal Chem 2009; 395: 283-300.

[43] Frank CD, Alan CS, Russell SH, Roy AW, Kevin LM, Aaron L, Raymond JW, Andrzej WM. Laser-induced breakdown spectroscopy (LIBS): A promising versatile chemical sensor technology for hazardous material detection. IEEE Sens J 2005; 5(4): 681-9.

[44] Jennifer LG, Frank CDL, Chase AM, Andrzej WM. Double-pulse stand-off laser-induced breakdown spectroscopy for versatile hazardous materials detection. Spectrochim Acta Part B 2007; 62: 1405-11.

[45] Whitehouse AI, Young J, Botheroyd IM, Lawson S, Evans CP, Wright J. Remote material analysis of nuclear power station steam generator tubes by laser-induced breakdown spectroscopy. Spectrochim Acta Part B 2001; 56: 821-30.

[46] Kuznitsov AV, Osetrov OI. Detection of improvised explosives and explosive devices. NATO Security through Science Series B: Physics and Biophysics, Proceedings of the NATO Advanced Research Workshop on Detection and Disposal of Improvised Explosives, St. Petersburg, Russia; In: Schubert H, Kuznetsov A. Eds.; 7-9 September 2005.

[47] Reinhard N, Cord FB. Stand-off detection of surface contaminations with explosives residues using laser-spectroscopic methods. Stand-off detection of suicide bombers and mobile subjects, NATO security through science series, In: Hiltmar S, Rimski-Korsakov A. Eds.; 2006; 89-99. 
[48] David SM, Jason SR. Portable Raman explosives detection. Anal Bioanal Chem 2009; 393:1571-1578.

[49] Pettersson A, Johansson I, Wallin S, Nordberg M, Ostmark H. Near real-time stand-off detection of explosives in a realistic outdoor environment at 55m distance. Propell Explos Pyrotech 2009; 34: 297306.

[50] Wiens RC, Sharma SK, Thompson J, Misra A, Lucey PG. Joint analyses by laser induced breakdown spectroscopy and Raman spectroscopy at stand-off distances. Spectrochim Acta A 2005; 61(10): 2324-34.

[51] Albert KJ, Myrick ML, Brown S, James D, Milanovich F, Walt DR. Field-deployable sniffer for 2,4-Dinitrotoluene detection. Environ Sci Technol 2001; 35: 3193-200.

[52] Albert KJ, Walt DR. High-speed fluorescence detection of explosives-like vapors. Anal Chem 2000; 72: 1947-1955.

[53] Orghici R, Willer U, Gierszewska M, Waldvogel S, Schade W. Fiber-optic evanescent field sensor for detection of explosives and CO2 dissolved in water. Appl Phys B: Lasers Opt 2008; 90: 35560.

[54] Schade W, Orghici R, Willer U, Waldvogel S. Fiber-optic evanescent-field sensor device for $\mathrm{CO} 2$ and explosive detection. In: Sampson D, Collins S, Oh K, Yamauchi R. Eds. 2008 19th International Conference on Optical Fibre Sensors, Proceedings of SPIE 2008; 7004: 700431-1.

[55] http://gs.flir.com/detection/explosives. September 4, 2013.
[56] Joseph DN, Device for testing surfaces of articles for traces of explosives and/or drugs. U. S. patent 7,456,393 B2, November 25, 2008.

[57] Williams VE, Lemieux RP, Thatcher G.RJ. Substituent effects on the stability of arene-arene complexes: An AM1 study of the conformational equilibria of cis-1,3-diphenylcyclohexanes. J Org Chem 1996; 61: 1927-1933.

[58] Yang JS, Swager TM. Fluorescent porous polymer films as TNT chemosensors: Electronic and structural effects. J Am Chem Soc 1998; 120: 11864-73.

[59] Dakin J, Culshaw B, Eds. Optical fiber sensors. Artech House Inc. 1997; 4: Ch. 7.

[60] Utzinger U, Richards-Kortum R. Fiber-optic probes for biomedical optical spectroscopy. J Bio Opt 2003; 8: 121-7.

[61] Plaza P, Quy DN, Jouan M, Fevrier H, Saisse H. Simulation et optimisation des capteurs à fibres optiques adjacentes. Appl Opt 1986; 25: 3448-54.

[62] www.oceanoptics.com. September 4, 2013.

[63] Nguyen H, Li X, Wang N, Wang Z, Ma J, Bock WJ, Ma D. Fiberoptic detection of explosives using readily available fluorescent polymers. Macromolecules 2008; 42: 921-6.

[64] Frank J.C, Hand held explosives detection system. U. S. Patent 5,138,889, August 18, 1990

[65] Craig AA., Colin JC., Mark EF, Michael JF, Marcus JL, Dennis KR., Mark GR, Eric ST, Vapor sensing instrument for ultra trace chemical detection. U. S. Patent 6,558,626 B1, May 6, 2003.

(C) Ma and Bock; Licensee Bentham Open.

This is an open access article licensed under the terms of the Creative Commons Attribution Non-Commercial License (http://creativecommons.org/licenses/by-nc/3.0/) which permits unrestricted, non-commercial use, distribution and reproduction in any medium, provided the work is properly cited. 